\title{
ARTICLE OPEN Alpl prevents bone ageing sensitivity by specifically regulating senescence and differentiation in mesenchymal stem cells
}

\author{
Wenjia Liu ${ }^{1,2}$, Liqiang Zhang ${ }^{1,2}$, Kun Xuan ${ }^{1}$, Chenghu Hu${ }^{2}$, Shiyu Liu ${ }^{1}$, Li Liao ${ }^{2}$, Bei Li ${ }^{1}$, Fang Jin ${ }^{1}$, Songtao Shi ${ }^{3}$ and Yan Jin ${ }^{1,2}$
}

Mutations in the liver/bone/kidney alkaline phosphatase (Alpl) gene cause hypophosphatasia (HPP) and early-onset bone dysplasia, suggesting that this gene is a key factor in human bone development. However, how and where Alpl acts in bone ageing is largely unknown. Here, we determined that ablation of $A / p l$ induces prototypical premature bone ageing characteristics, including bone mass loss and marrow fat gain coupled with elevated expression of p16 ${ }^{\text {INK4A }}$ (p16) and p53 due to senescence and impaired differentiation in mesenchymal stem cells (MSCs). Mechanistically, Alpl deficiency in MSCs enhances ATP release and reduces ATP hydrolysis. Then, the excessive extracellular ATP is, in turn, internalized by MSCs and causes an elevation in the intracellular ATP level, which consequently inactivates the AMPKa pathway and contributes to the cell fate switch of MSCs. Reactivating AMPKa by metformin treatment successfully prevents premature bone ageing in $\mathrm{Alpl}^{+/-}$mice by improving the function of endogenous MSCs. These results identify a previously unknown role of Alpl in the regulation of ATP-mediated AMPKa alterations that maintain MSC stemness and prevent bone ageing and show that metformin offers a potential therapeutic option.

Bone Research (2018)6:27; https://doi.org/10.1038/s41413-018-0029-4

\section{INTRODUCTION}

Alkaline phosphatase (ALP), which was initially identified in $1912{ }^{1}$ is a ubiquitous ectoenzyme widely distributed in nature from bacteria to humans. ${ }^{2}$ ALP is a well-known osteoblastic marker and has been widely used as a diagnostic index to evaluate bone formation capacity in osteoporosis. ${ }^{3,4}$ Clinically, a loss-of-function mutation in the liver/bone/kidney ALP (ALPL) gene has been linked to a severe skeletal deformity disease termed hypophosphatasia (HPP) that is characterized by bone mass loss and, consequently, pathological fractures. ${ }^{5-8} \mathrm{~A}$ genetic study investigating $A L P L$ in humans and mice strongly suggests that $A L P L$ is necessary for postnatal bone formation and that the bone deformities are related to the degree of $A L P L$ deficiency. ${ }^{5-8}$ Despite the established function of $A L P L$ in bone development, its role in bone ageing remains largely unknown.

Bone ageing, which is the main risk factor for primary osteoporosis, results in a decrease in bone mass and a parallel increase in marrow fat. ${ }^{2,9-11}$ At the cellular level, bone marrow (BM) mesenchymal stem cells (MSCs), which are common progenitors of osteoblasts (OBs) and adipocytes in the $\mathrm{BM}^{12,13}$ undergo senescence along with bone ageing. ${ }^{9,14,15}$ We and other scholars have provided evidence that rescuing the function of MSCs has a significant therapeutic impact on the accrual of the regeneration capacity and bone mass. ${ }^{16,17}$ Tissue nonspecific ALP (TNSALP), which is encoded by $A L P L$, is used as a surface marker for the prospective isolation of MSCs because TNSALP is enriched in the cell membrane. ${ }^{13,18}$ However, whether and how ALPL orchestrates the differentiation and senescence of MSCs and subsequently affects bone ageing remain elusive.
Adenosine triphosphate (ATP) has emerged as one of the most versatile extracellular molecules implicated in various cell processes ranging from energy supply to cell-to-cell signaling. ${ }^{19}$ As an ectonucleotidase, TNSALP appears to be involved in the metabolism of nucleotides and can sequentially hydrolyze ATP, ADP and AMP. ${ }^{20,21}$ Thus, the TNSALP level has been reported to be inversely correlated with the extracellular ATP concentration in neurocyte culture medium. MSCs and osteoblastic cell lineages have been shown to spontaneously release ATP, ${ }^{19,22,23}$ which plays a central role in bone physiology. ${ }^{24,25}$ Extracellular ATP is taken up by cells and leads to increased intracellular ATP to supplement the extra energy needs for growth, survival and apoptosis. ${ }^{26,27}$ Therefore, we hypothesize that Alpl may regulate ATP homeostasis in MSCs and subsequently lead to the fate switch in MSC differentiation and senescence.

In this study, we reveal that Alpl deficiency results in prototypical premature bone ageing characterized by bone mass loss and parallel marrow fat gain coupled with elevated p $16^{\text {INK4A }}$ (p16) and p53 expression. Here, our results suggest that bone ageing is partially orchestrated by Alpl, which regulates the differentiation and senescence of MSCs. Mechanistically, Alpl deficiency in MSCs results in enhanced ATP release and reduced ATP hydrolysis, which is, in turn, internalized by MSCs and consequently contributes to the cell fate change by regulating the AMPKa pathway. These data have far-reaching implications for the understanding of the role of $A l p l$ in bone ageing and the development of a new treatment method via the revaluation of Alpl.

\footnotetext{
'MS-State Key Laboratory \& National Clinical Research Center for Oral Diseases \& Shaanxi International Joint Research Center for Oral Diseases, Center for Tissue Engineering School of Stomatology, Fourth Military Medical University, Xi'an, China; ${ }^{2}$ Xi'an Institute of Tissue Engineering and Regenerative Medicine, Xi'an, China and ${ }^{3}$ Department of Anatomy and Cell Biology, School of Dental Medicine, University of Pennsylvania, Philadelphia, PA, USA

Correspondence: Yan Jin yanjin@fmmu.edu.cn, yanjinfmmu@139.com

These authors contributed equally: Wenjia Liu, Liqiang Zhang
}

Received: 5 February 2018 Revised: 16 June 2018 Accepted: 9 July 2018

Published online: 11 September 2018 


\section{RESULTS}

Decrease in $A l p l$ in the BM leads to bone ageing

Bone ageing is often manifested as a progressive decrease in bone mass and a parallel increase in marrow fat as shown by a microcomputer tomography $(\mu \mathrm{CT})$ analysis and Oil Red $\mathrm{O}$ staining (Supplementary Fig. 1a, b). Alpl is expressed in many organs and tissues, especially in the kidney, liver and bone, as shown in $\mathrm{Alp}^{\mathrm{Cre} /+}$; Rosa ${ }^{26 \mathrm{mTmG} /+}$ mice (Supplementary Fig. 2a). Notably, more $\mathrm{Alpl}^{+}$cells were observed in the BM than in the trabecular bone (Fig. 1a). Ageing-related p16 and p53 expression was elevated from adolescence (2 months) to advanced age (24 months) (Supplementary Fig. 1c). However, both ALP activity and TNSALP expression gradually declined with ageing (Fig. 1b-d) as indicated by the ALP activity assay, western blotting and immunohistochemical analyses. The inverse correlation was also recapitulated in a senescence-accelerated mouse (SAM) R1/P6 model (Supplementary Fig. 1d-h). Thus, using two ageing mouse models, we revealed that bone ageing characteristics could be associated with a decreased Alpl expression in the BM.

To further investigate the role of $A / p l$ in bone ageing, we injected $10 \mathrm{mg} \cdot \mathrm{kg}^{-1}$ levamisole, which is an ALP activity inhibitor, into the femoral BM cavity of 2-month-old mice every 2 weeks for 1 month (total of two injections), and $\mathrm{NaCl}$ was used as a control (Fig. 1e). The serum ALP activity was unchanged, but TNSALP expression in the BM progressively decreased 1 month after the injection (Fig. 1f, g). Notably, the inhibition of $A / p /$ expression induced more senescence-associated $\beta$-galactosidase activity (SA$\beta$-gal) ${ }^{+}$cells in the BM and higher expression levels of ageingrelated $p 16$ and $p 53$ (Fig. $1 \mathrm{~h}, \mathrm{i}$ ). The $\mu \mathrm{CT}$ analysis and Oil Red $\mathrm{O}$ staining showed that the bone mass was decreased, whereas the marrow adiposity was significantly increased compared to that in the non-injection group (Fig. $1 \mathrm{j}, \mathrm{k}$ ). These observations indicate that $\mathrm{Alpl}$ expression in the BM is inversely correlated with the progression of bone ageing.

Ablation of $A / p /$ results in premature bone ageing characteristics along with senescence and impaired differentiation of MSCs To further investigate the regulatory role of $A / p l$ in bone ageing, we used $A / p l-k n o c k o u t ~\left(C 57 B L / 6 J-A l p I^{+/-}\right.$) mice. In most cases, homozygous $\mathrm{Alpl}^{\gamma-}$ mice die at 20 days of age. ${ }^{28}$ Consequently, these mice are not appropriate for studying bone phenotype changes with age. In contrast, heterozygous $\mathrm{Alpl}^{+/-}$mice survive normally and show no changes in size, weight or appearance compared with wild-type (WT) littermates $(A / p)^{+/+}$) (Supplementary Fig. $2 \mathrm{~b}, \mathrm{c}$ ). A nearly $50 \%$ reduction in serum ALP activity was observed in 4-month-old $\mathrm{Alpl}{ }^{+/-}$mice relative to that in the WT group (Fig. 2a). The $\mu \mathrm{CT}$ analysis revealed that the $A /\left.p\right|^{+/-}$mice exhibited age-related bone loss compared with the WT controls at $4,8,12$ and 18 months as shown by a decreased BM density (BMD) and bone volume/total volume (BV/TV) (Fig. 2b, c). In contrast, compared with the WT controls, the BM adipose tissue gradually increased in the femur of the $\mathrm{Alpl}^{+/-}$mice as the animals aged as evidenced by Oil Red $\mathrm{O}$ staining (Fig. 2d, e). Moreover, fewer osteocalcin $(\mathrm{OCN})^{+}$cells but more peroxisome proliferatoractivated receptor $\gamma$ (PPAR $\gamma)^{+}$cells were observed in the BM of the $A / p I^{+/-}$mice (Fig. 2f), which is consistent with the perturbations in bone and adipose tissue in the $A / p I^{+/-}$mice.

In addition to the age-related bone loss and marrow fat gain in the $\mathrm{Alpl}^{+/-}$mice, the expression of $p 16$ was higher, whereas the expression of TERT declined in most organs of the 4-month-old $A / p I^{+/-}$mice, especially in tissues enriched in Alpl (Supplementary Fig. $2 d$, e). Specifically, the number of SA- $\beta-$ gal ${ }^{+}$cells and the expression of $p 16$ were obviously increased in the $A / p /^{+/-} \mathrm{BM}$, but TERT expression was decreased relative to that in the WT controls (Fig. $2 \mathrm{~g}$ and Supplementary Fig. 2d, e). Although the $\mathrm{Alp}^{+/-}$mice displayed age-related gradual bone loss, osteoclastogenesis in the $\mathrm{Alpl}^{+/-}$mice was almost the same as that in their WT controls as evidenced by the $\mu \mathrm{CT}$ analysis of cortical bone, tartrate-resistant acidic phosphatase (TRAP) staining and enzyme-linked immunosorbent assay (ELISA) analysis of carboxy-terminal telopeptide of type 1 collagen (CTX-1) (Supplementary Fig. 2g-i), indicating that Alpl-deficient bone displays premature ageing-related characteristics independent of osteoclastogenesis.

MSCs represent a promising cell source for the maintenance of bone homeostasis ${ }^{29,30}$ and undergo senescence with bone ageing. . $^{94,15}$ Interestingly, we observed that the $\mathrm{p} 16^{+}$senescent cells were mainly colocalized with $\mathrm{Sca}-1^{+}$cells but barely colocalized with $\mathrm{OCN}^{+}$cells in both the WT and $\mathrm{Alpl^{+/- }} \mathrm{BM}$ (Fig. 2h), indicating that the bone ageing phenotype of the $\mathrm{Alpl}^{+/-}$ mice may be related to the senescence of MSCs rather than OBs. To further validate this result, we tested the expression of $p 16$ and p53 in the WT and $\mathrm{Alpl}^{+/-} \mathrm{BM}$ sorted by flow cytometry as $\mathrm{CD} 73^{+} \mathrm{Sca} 1^{+} \mathrm{CD} 31^{-} \mathrm{CD} 45^{-}$. The increased $p 16$ and $p 53$ expression levels in the sorted MSCs were consistent with the results observed in the BM (Fig. 2i, j and Supplementary Fig. 2d, e). Taken together, these results suggest that the premature bone ageing characteristics caused by Alpl deficiency are probably due to the senescence and impaired differentiation of MSCs.

Alpl governs the osteo-adipogenic balance of MSCs and prevents cell senescence

To determine the role of $A / p l$ in MSC senescence and differentiation, we first compared the function of cultured MSCs between 4and 12-month-old WT and $A / p^{+/-}$mice. The MSCs were flushed from the BM and cultured in vitro (Supplementary Table 1). ALP activity and TNSALP expression were both decreased by more than half in the $\mathrm{Alpl}^{+/-}$MSCs compared with those in the WT MSCs (Fig. 3a). The Alpl ${ }^{+/-}$MSCs had relatively fewer $\mathrm{Ki}^{+} 7^{+}$cells and ageing-associated marker LAP $2 \beta^{+}$cells ${ }^{31}$ than the WT controls in the 4- and 12-month-old mice. However, many $\mathrm{Y}^{-\mathrm{H} 2 \mathrm{AX}}{ }^{+}$cells were observed, indicating that the DNA damage response was elevated, and SA- $\beta$-gal ${ }^{+}$cells and gradually increasing $p 16$ and $p 53$ expression were observed in the MSCs from the 4- and 12month-old $\mathrm{Alpl}^{+/-}$mice (Fig. 3b, c). In addition, the $\mathrm{Alpl}^{+/-} \mathrm{MSCs}$ manifested age-related enhanced adipogenic differentiation at the expense of osteogenic differentiation as shown by the decreased Runx2 and OCN levels and increased PPARy expression (Fig. 3d, e). These results indicate that Alpl deficiency in MSCs results in age-related senescence and impaired lineage differentiation.

To further validate the regulatory role of $A / p l$ in the fate switch of MSCs, we up- or downregulated the Alpl level in cultured MSCs via lentiviral transduction (Supplementary Fig. 3). The downregulation of $\mathrm{Alpl}$ in the WT MSCs significantly induced MSC senescence, whereas the recovery of $A l p l$ expression in the $\mathrm{Alpl}^{+/-}$ MSCs prevented cell senescence as evidenced by the expression of p16 and p53 (Fig. 3f). In addition, the inhibition of Alpl in the WT MSCs impaired their osteogenic differentiation but enhanced adipogenic differentiation, mimicking the $\mathrm{Alpl}^{+/-} \mathrm{MSC}$ phenotype. In contrast, the recovery of $\mathrm{Alpl}$ expression in the $\mathrm{Alpl}^{++-} \mathrm{MSCs}$ rescued their impaired osteogenic differentiation at the expense of adipogenic differentiation as confirmed by the Runx2, OCN and PPARY expression in vitro and the regeneration of new bone in vivo (Fig. $3 \mathrm{~g}-\mathrm{i}$ ). More convincingly, the increased expression of Alpl in the WT MSCs partially prevented the senescence caused by a high concentration of $\mathrm{H}_{2} \mathrm{O}_{2}$ and recovered their osteoadipogenic differentiation (Fig. $3 \mathrm{j}-\mathrm{l}$ ). Therefore, Alpl is necessary for lineage alteration in MSCs and prevents their senescence, subsequently affecting the phenotype of postnatal bone.

Alpl deficiency induces the release of ATP, which is, in turn, internalized by MSCs and causes their dysfunction

Given that TNSALP has been reported to hydrolyze inorganic pyrophosphate (PPi) to promote OB mineralization, ${ }^{32}$ we investigated whether TNSALP regulates the differentiation of MSCs through PPi. The extracellular PPi concentrations were indeed 

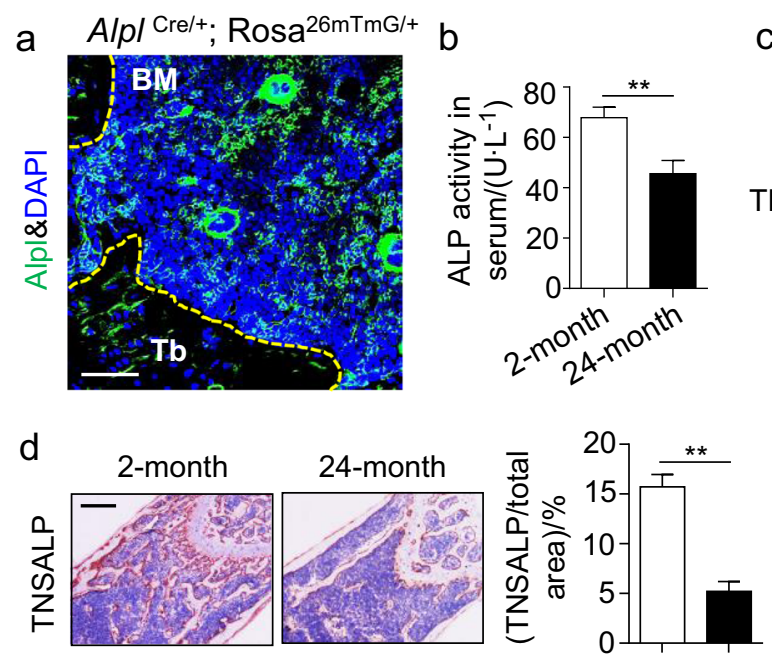

$2-m \frac{n+m}{24 m}+m+n$

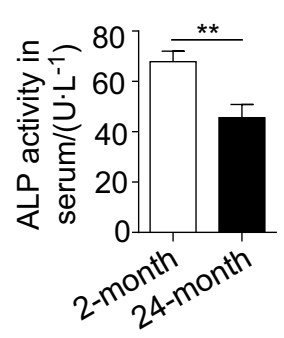

C
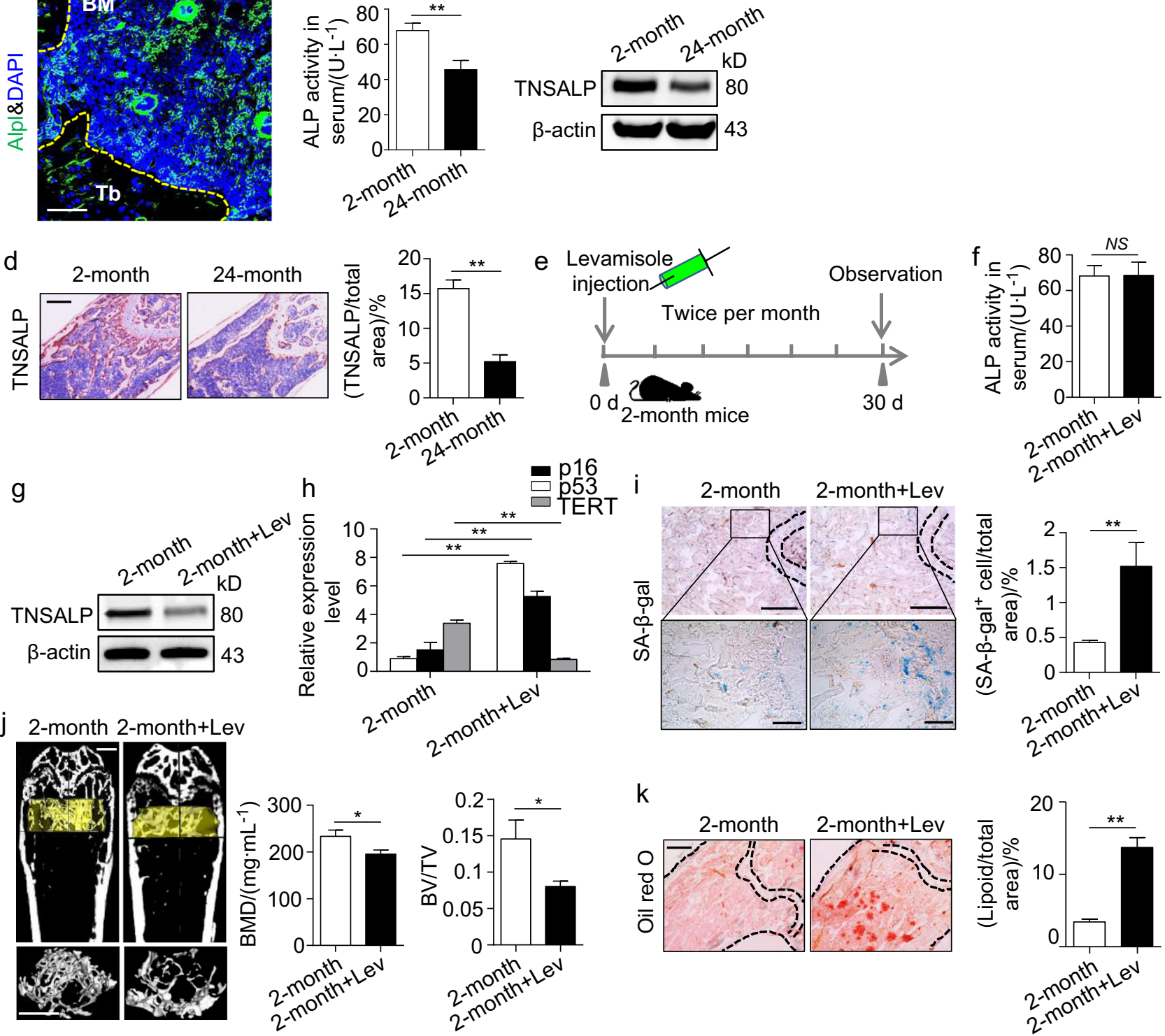

$\mathrm{k}$
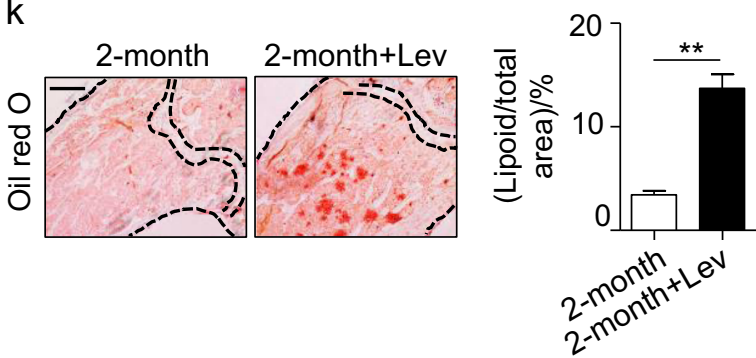

Fig. 1 Declined expression of $\mathrm{Alpl}$ in the bone marrow is associated with bone ageing characteristics. a Representative image of immunostaining analysis showing $\mathrm{Alpl}$ (green) in the BM of 2 -month-old $\mathrm{Alpl}{ }^{\mathrm{Cre} /{ }^{+}}$; Rosa ${ }^{26 \mathrm{mTmG} /+}$ mice. Scale bar: $50 \mu \mathrm{m}$. b, c ALP activities in serum and expression levels in the BM of young (2-month) and old (24-month) mice were analyzed by an ALP activity assay and a western blotting analysis. d Immunohistochemical analysis of TNSALP (brown) of the femoral diaphysis. Quantification of the TNSALP ${ }^{+}$area is indicated on the right. Scale bars, $500 \mu \mathrm{m}$. e Scheme of the levamisole injection mouse model. We injected $10 \mathrm{mg} \cdot \mathrm{kg}^{-1}$ levamisole into the femoral bone marrow cavity of 2-month-old mice every 2 weeks for 1 month (total of two injections), and NaCl was used as a control. f, $\mathbf{g}$ ALP activities and expression levels were analyzed by an ALP activity assay and a western blotting analysis. $\mathbf{h}$ Expression levels of the ageingspecific genes p16, p53 and TERT were examined via qRT-PCR. i SA- $\beta$-gal staining in the BM and quantification of $\beta$-gal ${ }^{+}$(blue) area are indicated on the right. Scale bars, $500 \mu \mathrm{m}$ (upper). Scale bars, $100 \mu \mathrm{m}$ (lower). $\mathbf{j} \mu C T$ images and quantification of bone mineral density (BMD) and bone volume/total volume (BV/TV) of the femur. Scale bars, $1 \mathrm{~mm}$. $\mathbf{k}$ Oil Red $\mathrm{O}$ staining images and quantitative analyses of the area of adipose tissue over the total area of the femoral diaphysis. Scale bars, $500 \mu \mathrm{m}$. Young group, $n=6$, Old group, $n=6$; all injection groups, $n=8$. The data are presented as the means \pm standard deviation (s.d.) of each experiment performed in triplicate. ${ }^{*} P<0.05,{ }^{*} P<0.01, N S$ not significant. Unpaired two-tailed Student's $t$-test

varied according to the Alpl level, but the intracellular PPi levels were unaffected. Additionally, exogenous PPi merely inhibited the osteogenic differentiation of MSCs but barely affected their adipogenic differentiation (Supplementary Fig. 4), suggesting that PPi is not a major factor in the MSC lineage alteration caused by Alpl.

TNSALP is also an efficient ATP ectonucleotidase, ${ }^{33,34}$ and extracellular ATP has been reported to regulate the proliferation, migration and differentiation of MSCs. ${ }^{35}$ Therefore, we tested whether ATP was involved in the Alpl-mediated cell fate choice of MSCs. As predicted, the extracellular ATP level in the Alp/ ${ }^{+/-}$MSCs was significantly higher than that in the WT MSCs (Fig. 4a). MSCs have been shown to spontaneously release ATP, ${ }^{19,22}$ and the major release mechanism is through tissue and cell damage. ${ }^{19,25}$ However, we observed that apoptosis in the $\mathrm{Alpl}^{+/-} \mathrm{MSCs}$ was identical to that in the WT MSCs (Fig. 4b), indicating that the higher extracellular ATP level in the Alpl-deficient MSCs may not be caused by cell death. Therefore, we hypothesized that Alpl may 

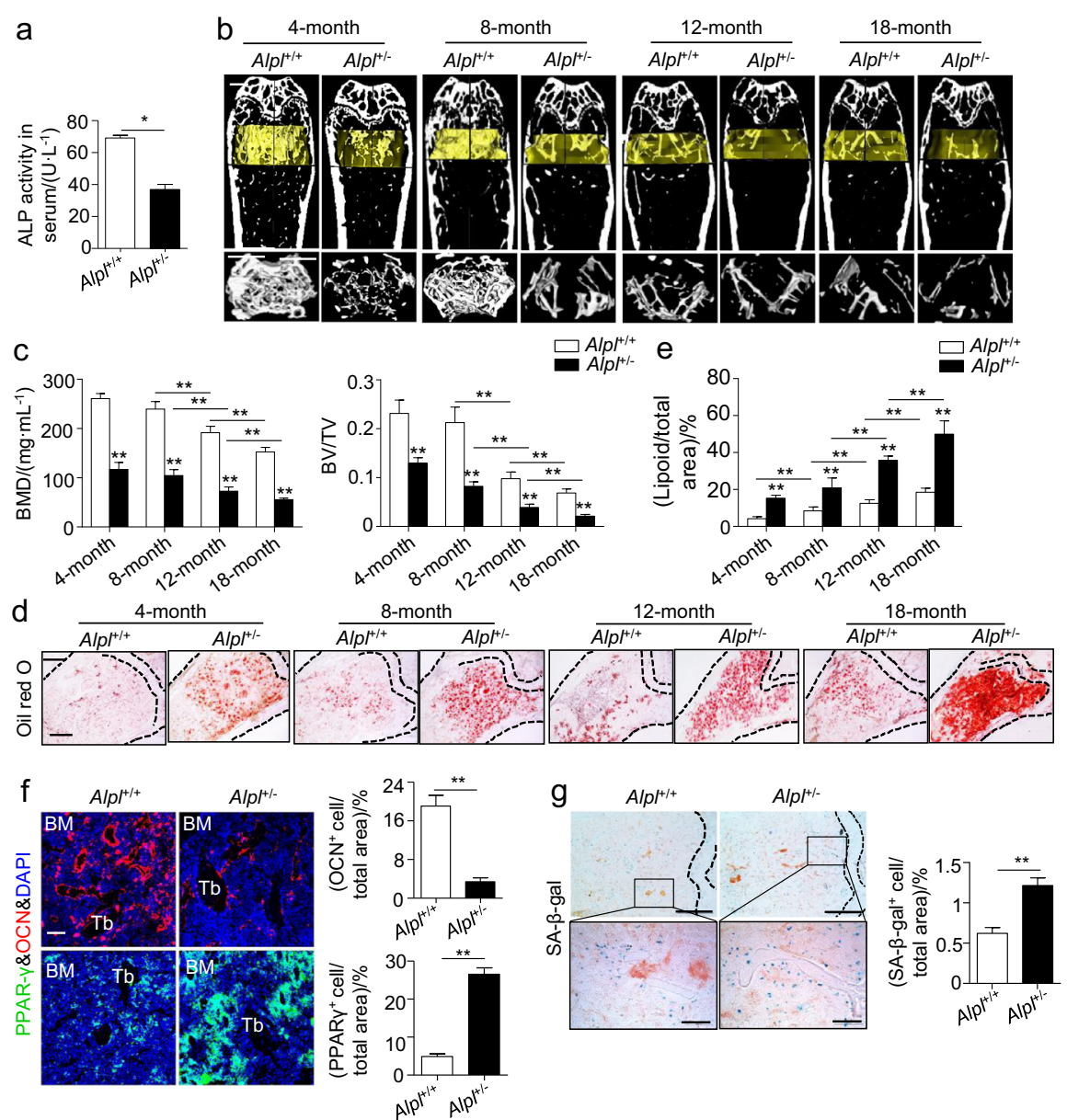

h Sca-1\&DAPI

p16\&DAPI
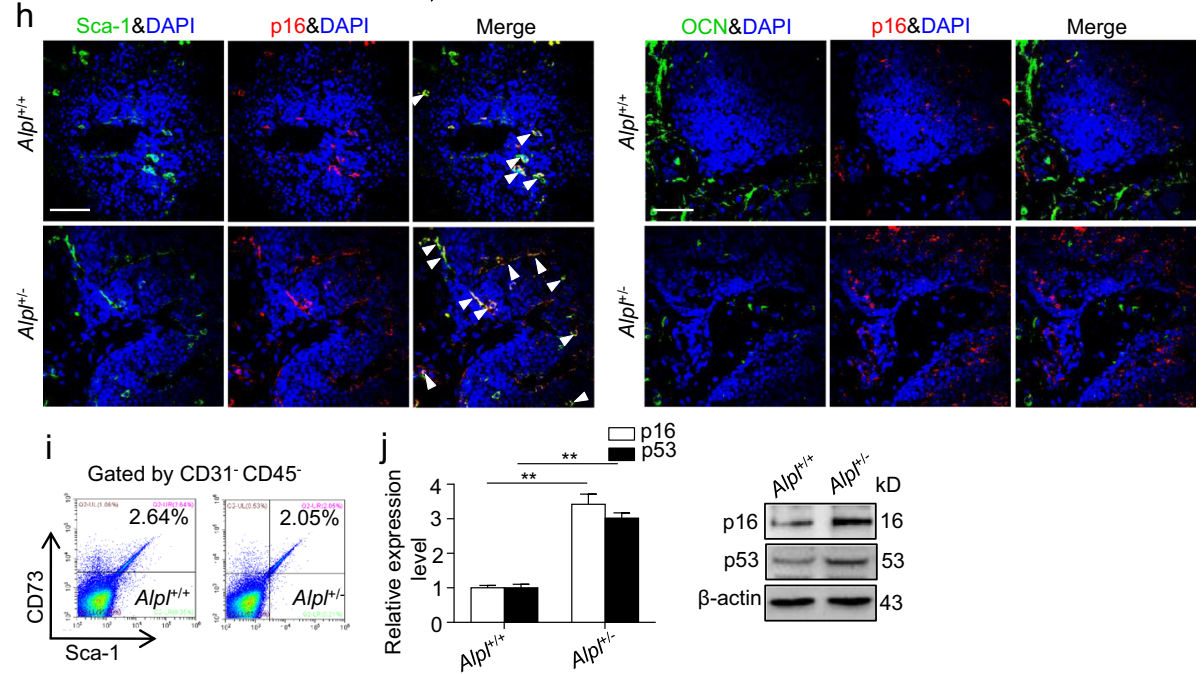

Fig. 2 Heterozygous $A /\left.p\right|^{+/-}$mice exhibit age-related bone mass loss and marrow fat gain, mimicking premature bone ageing. a Serum ALP activities in 4-month-old $A / p I^{+/+}$and $A /\left.p\right|^{+/-}$mice were analyzed by an ALP activity assay. $\mathbf{b}, \mathbf{c} \mu \mathrm{CT}$ images and quantification of BMD and BV/TV. Scale bars, $1 \mathrm{~mm}$. d, e Oil Red $\mathrm{O}$ staining images and quantitative analysis of the area of adipose tissue over the total area of the proximal femoral diaphysis. Scale bars, $500 \mu \mathrm{m}$. $\mathbf{f}$ Immunostaining analysis of OCN (red), PPAR- $\gamma$ (green) and nuclear staining (blue, DAPI) of the proximal femoral diaphysis. Quantification of $\mathrm{OCN}^{+}$and PPAR- $\gamma^{+}$cells is indicated in the right panel. Scale bars, $200 \mu \mathrm{m}$. g SA- $\beta-\mathrm{gal}$ staining in the BM and quantification of the $\beta$-gal ${ }^{+}$area are indicated on the right. Scale bars, $500 \mu \mathrm{m}$ (upper). Scale bars, $100 \mu \mathrm{m}$ (lower). $\mathbf{h}$ Immunostaining showed overlap between p16 (red) and Sca-1/OCN (green) in the $\mathrm{Alpl} l^{+/+}$and $\mathrm{Alpl}{ }^{+/-} \mathrm{BM}$. The white arrows indicate the p16 ${ }^{+} \mathrm{Sca}-1^{+} \mathrm{cells}$. Scale bar: $100 \mu \mathrm{m}$. i Representative dot plot of MSC FASC analysis of the expression $(+)$ or absence $(-)$ of the following standard markers: $\mathrm{CD} 73^{+} \mathrm{SCa} 1^{+} \mathrm{CD} 45^{-} \mathrm{CD} 31^{-}$. j Expression levels of the ageing-specific genes $p 16$ and $p 53$ in FASC sorted cells were examined via qRT-PCR and western blotting. $n=6$ per group. The data are presented as the means \pm s.d. of each experiment performed in triplicate. ${ }^{*} P<0.05$, ${ }^{*} P<0.01$, unpaired two-tailed Student's $t$-test 
a

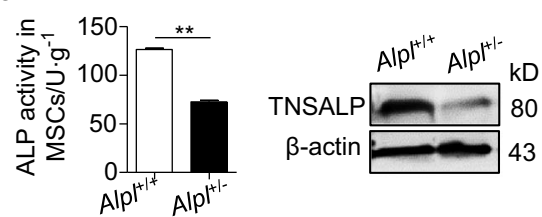

d
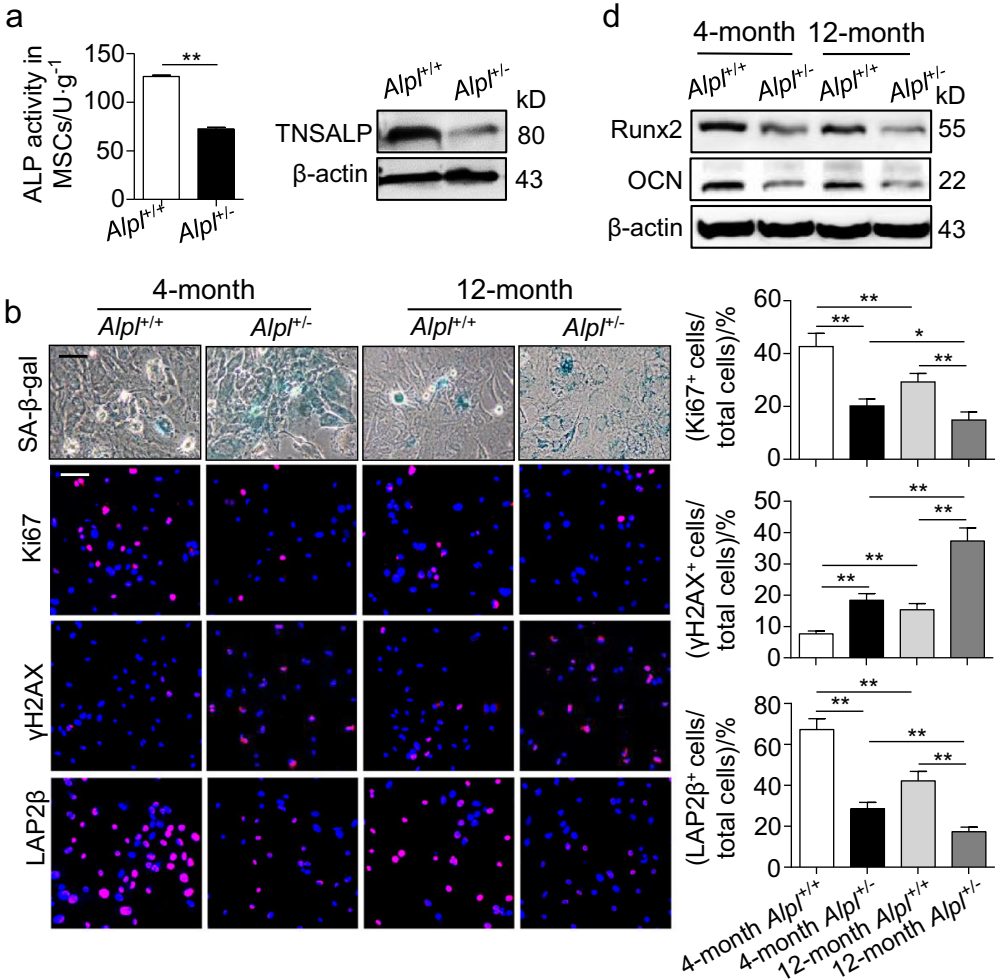

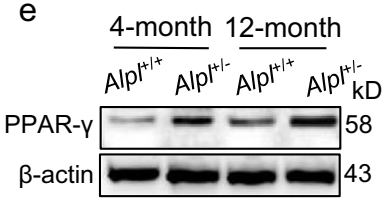

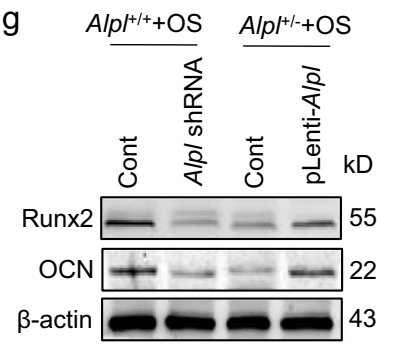
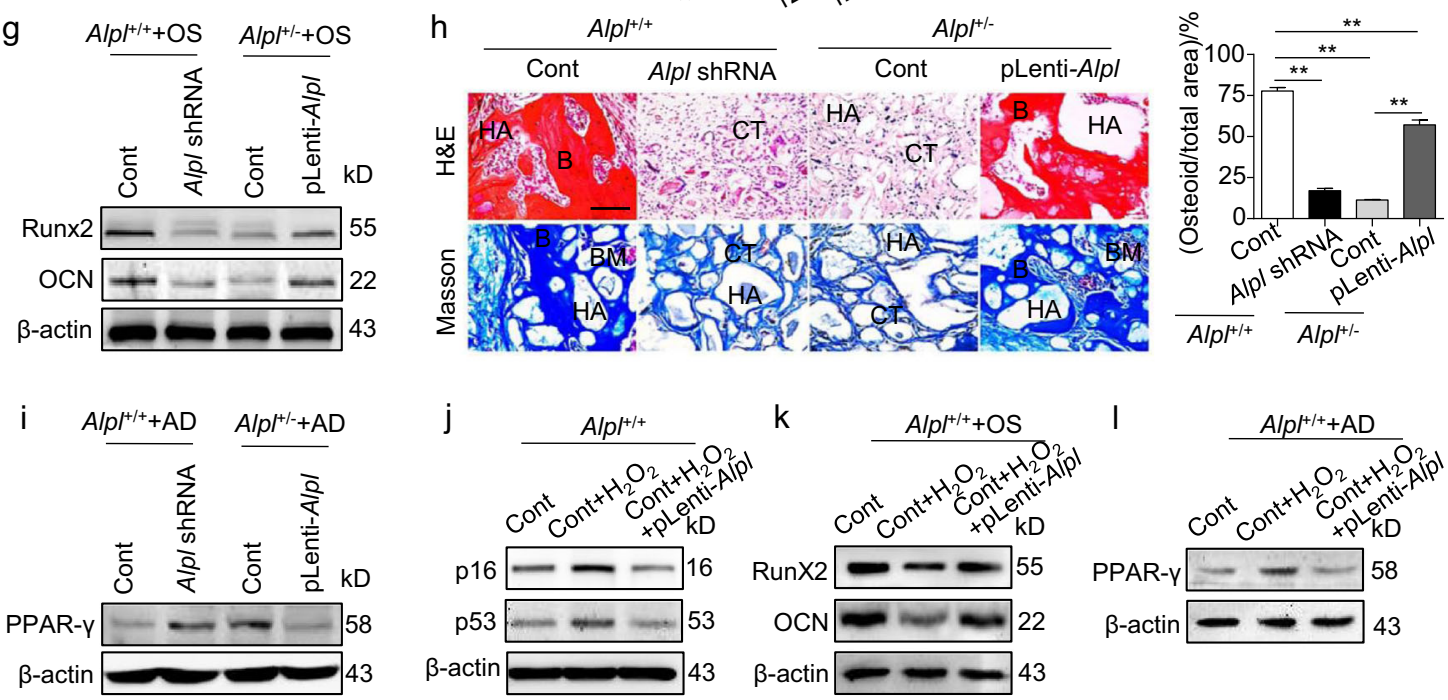

Fig. 3 Alpl controls the osteo-adipogenic balance in MSCs and prevents their senescence. a ALP activities and expression levels in Alp/ $^{+/+}$and $A / p 1^{+/-}$MSCs were examined by an ALP activity assay and a western blotting analysis. b SA- $\beta$-gal staining and Ki67, $\gamma \mathrm{H} 2 \mathrm{AX}$ and LAP2 $\beta$ immunostaining of first-passage MSCs from Alpl ${ }^{+/+}$and $A / p l^{+/-}$mice at 4 and 12 months. Quantification of $\mathrm{Ki}^{+} 7^{+}, \gamma \mathrm{H} 2 \mathrm{AX}{ }^{+}$and $\mathrm{LAP}_{2} \beta^{+}$is shown in the right panel. Scale bars: $50 \mu \mathrm{m}$. c Expression levels of p16 and p53 in MSCs from $A / p I^{+/+}$and $A / p I^{+/-}$mice at 4 and 12 months were examined by a western blotting analysis. d, e Expression levels of Runx2, OCN and PPAR- $\gamma$ in MSCs from Alp/ $I^{+/+}$and $A / p I^{+/-}$mice at 4 and 12 months were examined by a western blotting analysis on day 7 after the osteogenic/adipogenic induction. $f$ Downregulated Alpl

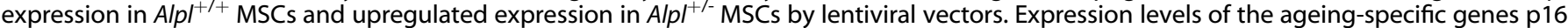
and $\mathrm{p} 53$ in MSCs were examined by a western blotting analysis. g Expression levels of Run 2 and OCN were examined by a western blotting analysis on day 7 after the osteogenic induction. $\mathbf{h} \mathrm{HE} /$ Masson's trichrome staining and quantitative analysis revealed the formation of bone (B), bone marrow (BM) and collagen fiber (CF) around the HA/TCP (HA) carrier after the MSCs were implanted into nude mice. Scale bars, 200 $\mu \mathrm{m}$. i PPAR- $\gamma$ expression was examined on day 7 after the adipogenic induction by western blotting. $\mathbf{j}$ Alp/ ${ }^{+/+} \mathrm{MSCs}$ were treated with $50 \mu \mathrm{M}$ $\mathrm{H}_{2} \mathrm{O}_{2}$ for $24 \mathrm{~h}$ with or without the overexpression of $\mathrm{Alpl}$; then, the medium was replaced with normal medium, followed by incubation for another $24 \mathrm{~h}$. Expression levels of p16 and p53 were examined by a western blotting analysis. $\mathbf{k}, \mathrm{I}$ Alp ${ }^{+/+} \mathrm{MSCs}$ were treated with $50 \mu \mathrm{M} \mathrm{H} \mathrm{H}_{2} \mathrm{O}_{2}$ for $24 \mathrm{~h}$ with or without $A / p l$ overexpression, and then, the medium was changed to induction medium, followed by incubation for $7 \mathrm{~d}$. Expression levels of Runx2, OCN and PPAR $\gamma$ were examined by a western blotting analysis. $n=6$ per groups. The data are shown as the means \pm s.d. of each independent experiment performed in triplicate. ${ }^{*} P<0.05,{ }^{* *} P<0.01$. a Unpaired two-tailed Student's $t$-test. $\mathbf{b}$, $\mathbf{h}$ One-way analysis of variance (ANOVA) 


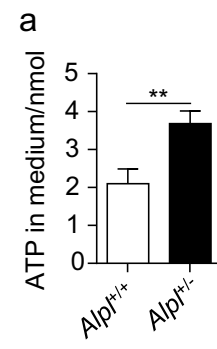

b
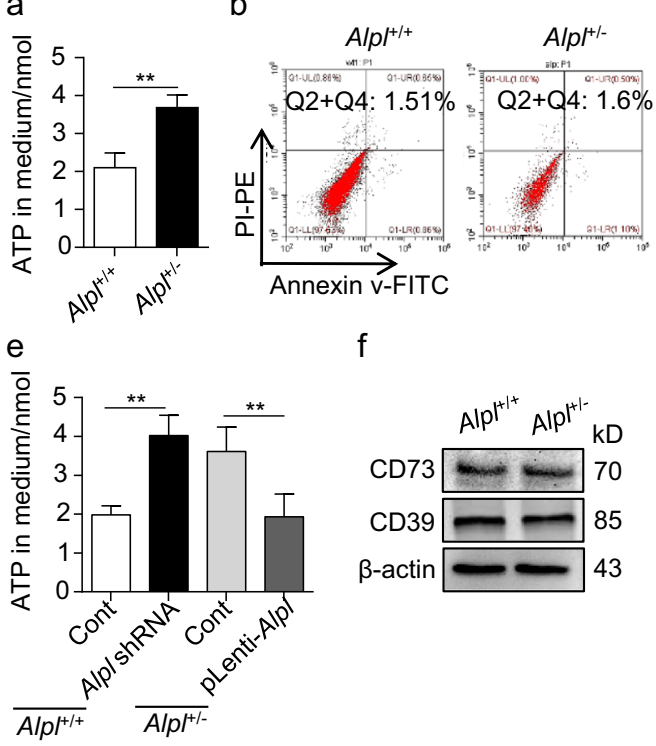

f
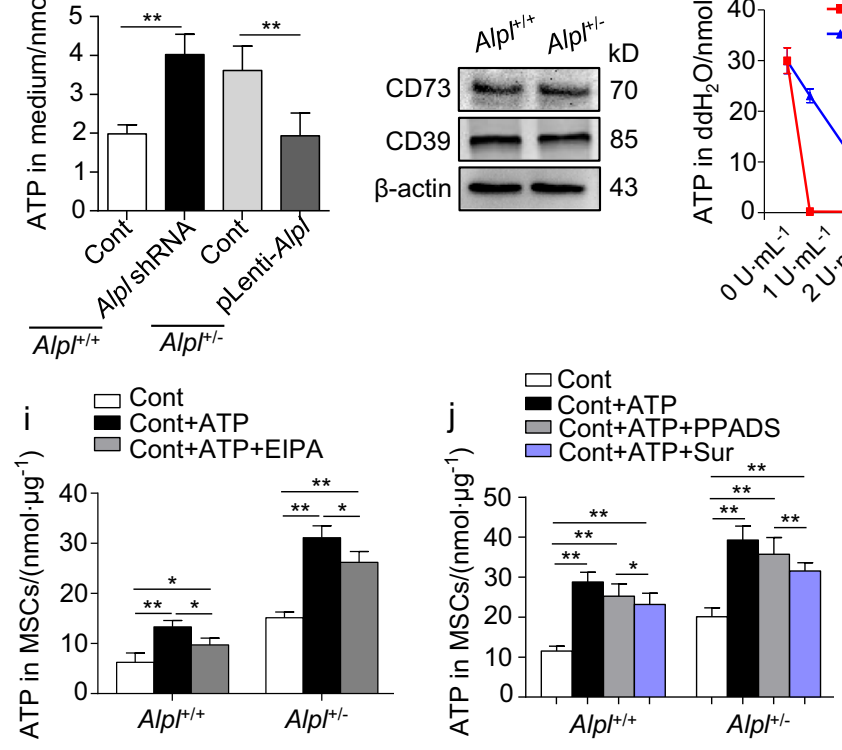

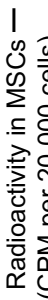

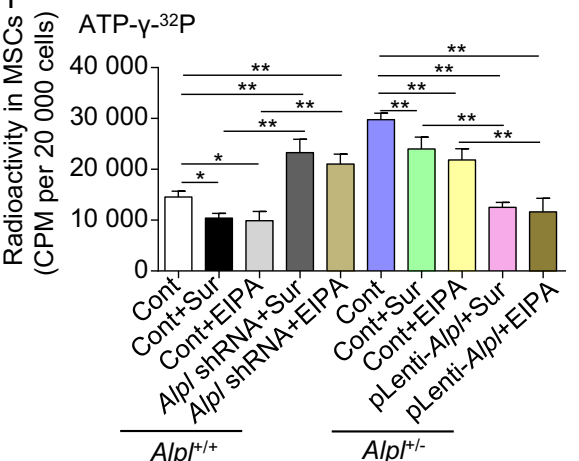

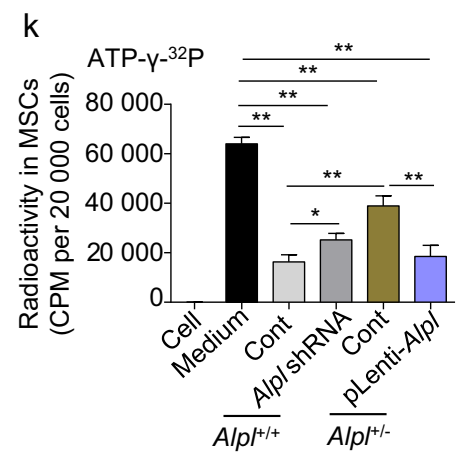

$\mathrm{m}$

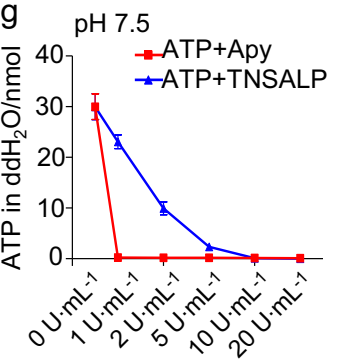

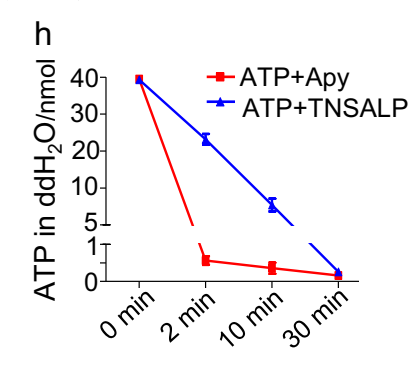
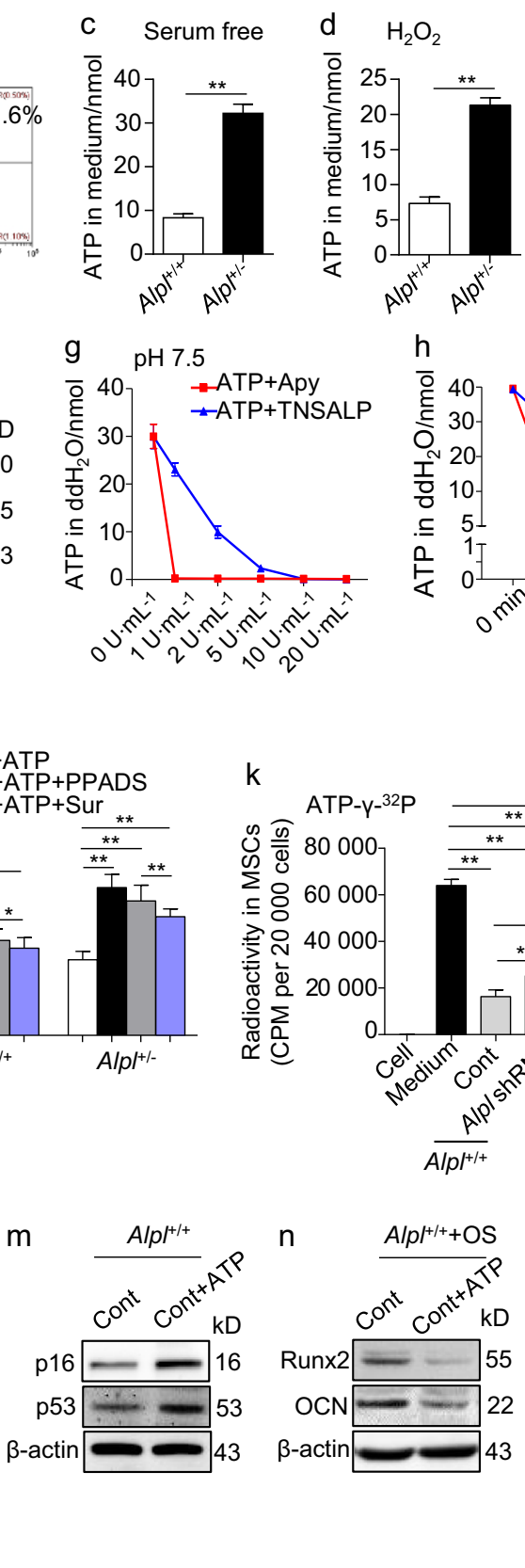

Fig. 4 Alpl deficiency induces an elevation in extracellular ATP, which is internalized by MSCs and causes their dysfunction. a Extracellular ATP concentrations in $\mathrm{Alpl}^{+/+}$and $\mathrm{Alpl}^{+/-} \mathrm{MSC}$ medium were assayed by a regular ATP concentration assay. b Apoptosis of $\mathrm{Alpl}^{+/+}$and $\mathrm{Alpl^{+/- }} \mathrm{MSCs}^{-1}$ was analyzed by flow cytometry. c, d Extracellular ATP concentrations were assayed $1 \mathrm{~h}$ after $\mathrm{FBS}$ deprivation and $\mathrm{H}_{2} \mathrm{O}_{2}$ induction $\left(50 \mathrm{mmol} \cdot \mathrm{L}^{-1}\right)$. e Extracellular ATP concentrations were assayed $48 \mathrm{~h}$ after transduction with different lentiviral vectors. $\mathrm{f}$ Expression levels of CD73 and CD39 in $\mathrm{Alpl}^{+/+}$and $\mathrm{Alpl^{+/- }}$ MSCs were analyzed by western blotting. g ATP concentrations were assayed after 2 min of treatment with $0,1,2,5,10$ or $20 \mathrm{U} \cdot \mathrm{mL}^{-1}$ of TNSALP and $2 \mathrm{U} \cdot \mathrm{mL}^{-1}$ of ATP-apyrase in the presence of $20 \mathrm{nmol} \cdot \mathrm{L}^{-1}$ ATP in double-distilled water (dd $\mathrm{H}_{2} \mathrm{O}$, $\mathrm{pH}$ 7.5). $\mathbf{h}$ ATP concentrations were assayed at $0 \mathrm{~min}, 2 \mathrm{~min}, 10 \mathrm{~min}$ and $30 \mathrm{~min}$ after treatment with $10 \mathrm{U} \cdot \mathrm{mL}^{-1}$ of TNSALP and $2 \mathrm{U} \cdot \mathrm{mL}^{-1}$ of ATPapyrase in the presence of $20 \mathrm{nmol} \cdot \mathrm{L}^{-1}$ ATP in dd $\mathrm{H}_{2} \mathrm{O}$. $\mathbf{i}, \mathbf{j}$ Alp/ ${ }^{+/+}$and $A / p l^{+/-} \mathrm{MSC}$ were treated with $10 \mu \mathrm{mol} \cdot \mathrm{L}^{-1}$ ATP in the presence or absence of $50 \mu \mathrm{mol} \cdot \mathrm{L}^{-1}$ ethyl isopropyl amiloride (EIPA), $30 \mu \mathrm{mol} \cdot \mathrm{L}^{-1}$ pyridoxal phosphate-6-azo (PPADS) or $100 \mu \mathrm{mol} \cdot \mathrm{L}^{-1} \mathrm{suramin}(\mathrm{Sur})$, and the intracellular ATP concentrations were assayed $1 \mathrm{~h}$ after treatment. $\mathbf{k}$ Intracellular radioactivity was examined after a 1 - $\mathrm{h}$ treatment with ATP$\gamma^{-32} \mathrm{P}$ in the different lentivirus transduction groups. I Intracellular radioactivity was examined after a $1-\mathrm{h}$ treatment with ATP- $\gamma^{-32} \mathrm{P}$ in different lentivirus transduction groups treated with $100 \mu \mathrm{mol} \cdot \mathrm{L}^{-1}$ suramin and $50 \mu \mathrm{mol} \cdot \mathrm{L}^{-1}$ EIPA. $\mathbf{m}, \mathbf{n ~ A l p l ^ { + / + }} \mathrm{MSCs}$ were treated with $10 \mu \mathrm{mol} \cdot \mathrm{L}^{-1} \mathrm{ATP}$, and the ageing-specific genes were analyzed after $48 \mathrm{~h}$. Expression levels of Runx2, OCN and PPAR were examined by western blotting on day 7 after induction. $n=6$ per group. The data are presented as the means \pm s.d. of each independent experiment performed in triplicate. ${ }^{*} P<0.05,{ }^{*} P<0.01$. a-d, $\mathbf{g}-\mathbf{h}, \mathbf{i}$-j Unpaired two-tailed Student's $t$-test. e, $\mathbf{k}$, I One-way analysis of variance (ANOVA) 
regulate ATP release. To confirm this hypothesis, we induced MSCs by serum starvation and $\mathrm{H}_{2} \mathrm{O}_{2}$ treatment. After the induction, the $\mathrm{Alpl}^{+/-} \mathrm{MSCs}$, but not the WT MSCs, released a large amount of ATP (Fig. 4c, d). More strikingly, the downregulation of Alpl led to increased extracellular ATP in the WT MSCs, whereas enforcing expression in the $\mathrm{Alpl}^{+/-} \mathrm{MSCs}$ significantly reduced the extracellular ATP level (Fig. 4e), suggesting that Alpl in MSCs probably regulates ATP release.

Nucleoside triphosphate dephosphorylase (CD39) and ecto-5'nucleotidase (CD73) are also classical cell surface ATP apyrases that hydrolyze ATP/ADP to AMP and adenosine, respectively. ${ }^{27,36}$ However, in contrast to the expression of TNSALP, the expression of CD39 and CD73 did not significantly differ between the WT and $\mathrm{Alpl}^{+/-}$MSCs (Fig. 4f). Additionally, in contrast to the classical ATPapyrase, the enzymatic reaction experiments revealed that the hydrolysis of TNSALP occurred in a dose- and time-dependent manner (Fig. $4 \mathrm{~g}, \mathrm{~h}$ ). Due to the decrease in TNSALP activity and expression by nearly $50 \%$ in the $\mathrm{Alpl}^{+/-}$MSCs (Fig. 3a), their ATP hydrolytic ability was probably weaker than that of the WT MSCs, further resulting in a higher extracellular ATP level. Therefore, coupled with more ATP release from $\mathrm{Alpl}^{+/-} \mathrm{MSCs}$, the extracellular ATP level was further increased due to the Alpl deficiency.

We further noticed that similar to the extracellular ATP level, the intracellular ATP concentrations were changed according to the Alpl level (Supplementary Fig. 5a-c). Additionally, this elevation due to the Alpl deficiency was independent of ATP synthesis (Supplementary Fig. 5d). Thus, we investigated how extracellular ATP affects the intracellular ATP level and, subsequently, cell function. The intracellular ATP concentration in the cells treated with ATP and ethyl isopropyl amiloride (EIPA), which is a macropinocytosis inhibitor, ${ }^{37,38}$ was only reduced by nearly $16 \%-20 \%$ compared with that in the group that was only treated with ATP but was still 70\% higher than the baseline level in the control group (Fig. 4i), indicating that macropinocytosis is not a major determinant of the intracellular ATP increase. Additionally, previous studies have reported that extracellular ATP regulates cell function by activating ionotropic P2X and metabotropic P2Y receptors. $^{39-41}$ Thus, we blocked these receptors with pyridoxal phosphate-6-azo (PPADS) and suramin, which are nonselective P2X and P2Y receptor antagonists, respectively. Similar to the EIPA experiment, the ATP levels were decreased by no $>20 \%$ in both the WT and $\mathrm{Alpl}^{+/-}$MSCs treated with PPADs and suramin (Fig. 4j), indicating that the intracellular ATP levels in MSCs are not mainly affected by traditional purinergic signaling.

To further elucidate how the intracellular ATP levels were affected by the extracellular ATP levels, we performed radioisotope experiments. The results showed that the intracellular radioactivity of ATP- $\mathrm{Y}^{-}{ }^{32} \mathrm{P}$ was $2.5-$ fold higher in the $\mathrm{Alp} \mathrm{I}^{+/-} \mathrm{MSCS}$ than that in the WT MSCs after adding radioactive ATP to the cell culture medium for $1 \mathrm{~h}$. Furthermore, the intracellular radioactive profile was inversely correlated with the Alpl level (Fig. 4k and Supplementary Fig. 5e). More importantly, the radioactivity could not be blocked by either the EIPA or suramin treatment, which is consistent with the regular ATP assays (Fig. 4l). As predicted, exogenous ATP added to the WT MSC culture medium could cause increased intracellular ATP that not only induced senescence but also inhibited osteogenesis, promoting adipogenesis (Fig. 4m, n). Collectively, these findings suggest that Alpl deficiency induces the excessive elevation of extracellular ATP, which is subsequently internalized by MSCs mainly by directly entering the cytoplasm and causing their fate changes.

ATP induces MSC dysfunction by repressing the AMPKa pathway Subsequently, we aimed to obtain insight into how ATP regulates the function of MSCs. The AMP-activated protein kinase a (AMPKa) pathway is directly regulated by the ATP level. ${ }^{42}$ We observed that the addition of ATP to the culture medium of WT MSCs caused an increase in intracellular ATP, thereby inhibiting the phosphorylation of AMPKa-Thr172 (p-AMPKa), which peaked 1 and $3 \mathrm{~h}$ after incubation (Fig. 5a). Thus, we investigated the relationship between the AMPKa pathway and Alpl level. As expected, p-AMPKa, but not total AMPKa, was significantly decreased in the $\mathrm{Alpl}^{+/-} \mathrm{MSCs}$ (Fig. 5b). More convincingly, the phosphorylation of AMPKa and its downstream target acetyl-CoA carboxylase (ACC) were correlated with the level of Alpl, whereas the total protein did not change as indicated by a western blotting analysis (Fig. 5c). Importantly, this ATP-mediated inactivation of the AMPKa pathway was not rescued by the suramin or EIPA treatment (Fig. 5d). To further validate our observations, we collected the culture medium of WT and Alp/ ${ }^{+/-}$MSCs. The supernatant of the $A / \mathrm{pl}^{+/-} \mathrm{MSC}$ suppressed the expression levels of p-AMPKa and p-ACC in 293T cell lines (Fig. 5e), indicating that the higher extracellular ATP levels due to the Alpl deficiency could inactivate the AMPKa pathway.

The AMPKa pathway also plays a role in bone physiology. The activation of AMPK promotes bone formation in vitro, and the deletion of the $a$ or $\beta$ subunit of AMPK decreases bone mass in mice. ${ }^{43,44}$ We also observed that the inhibition of the AMPKa pathway in MSCs promoted adipogenic differentiation at the expense of osteogenic differentiation (Supplementary Fig. 6). More strikingly, the addition of ATP could not induce senescence and impair the lineage differentiation of WT MSCs after the knockdown of AMPKa by small interfering RNA (siRNA) (Fig. 5f-h). Taken together, these data demonstrate that ATP directly represses the AMPKa pathway, thereby contributing to the alterations in the differentiation and senescence of MSCs.

Metformin rescues endogenous MSC function and prevents premature bone ageing in $\mathrm{Alpl}^{+/-}$mice

As Alpl and the AMPKa pathway both effectively regulate the function of MSCs, we compared the therapeutic effect of Alpl overexpression and AMPKa activation on the recovery of $\mathrm{Alpl}^{+/-}$ MSCs. Metformin is a common activator of the AMPKa pathway ${ }^{3}$ and has been recently used as an anti-ageing drug. ${ }^{45}$ Notably, metformin exhibited a stronger capacity to rescue the differentiation of $\mathrm{Alpl}^{+/-} \mathrm{MSC}$ than Alpl expression as evidenced by the increased osteogenic differentiation and decreased adipogenic differentiation (Supplementary Fig. 7a, b). In addition, compared with the $\mathrm{Alpl}^{+/-}$mice in the non-treated groups, the skeletal deformities in the $\mathrm{Alpl}^{+/-}$mice given metformin-treated $\mathrm{Alpl}^{+/-}$ MSCs were ameliorated (Supplementary Fig. 7c, d).

To determine whether metformin exerted a therapeutic effect in vivo, we injected $60 \mathrm{mg} \mathrm{kg}^{-1}$ metformin into the femoral BM cavity of 4-month-old $\mathrm{Alpl}^{+/-}$mice twice per month for 1 month, and $0.9 \% \mathrm{NaCl}$ was used as the vehicle and control. The expression levels of p-AMPKa and p-ACC were elevated in the Alp/ ${ }^{+/-}$MSCs after the metformin injections (Fig. 6a). Consequently, more Ki67 ${ }^{+}$ cells and $\mathrm{LAP}_{2} \beta^{+}$cells but fewer $\mathrm{YH}_{2} \mathrm{AX}{ }^{+}$cells were observed in the injection group compared with those in the non-treatment group (Fig. 6b). Moreover, a declined $p 16$ and $p 53$ expression was also detected in the metformin-treated group (Fig. 6c), indicating that the senescence characteristics of the endogenous $\mathrm{Alpl}^{+1-}$ MSCs were rescued by the metformin treatment. Along with the senescence recovery in the $\mathrm{Alpl}^{+/-} \mathrm{MSCs}$, lineage differentiation was rescued by metformin as evidenced by an enrichment in mineralized nodules, increased expression levels of Runx2 and OCN, and reduced fat depots and PPARy expression (Fig. 6d, e). Importantly, we observed that metformin restored p-AMPKa expression in the $\mathrm{BM}$ of the $\mathrm{Alpl}^{+/-}$mice (Fig. 6f). Thus, the premature bone ageing in the $\mathrm{Alpl}^{+/-}$mice was attenuated, representing a 1.9-fold BMD, 1.9-fold bone formation rate/bone surface (BFR/BS) and 2.5-fold BV/TV improvement compared with those in the $\mathrm{NaCl}$ group (Fig. $6 \mathrm{~g}, \mathrm{~h}$ ). In contrast, both the adipose tissue and expression of $p 16$ and $p 53$ in the $\mathrm{BM}$ of the $\mathrm{Alpl}^{+/-}$mice were reduced by $>50 \%$ after the metformin treatment (Fig. $6 i, j$ ). Notably, the therapeutic effect of metformin on rescuing the 


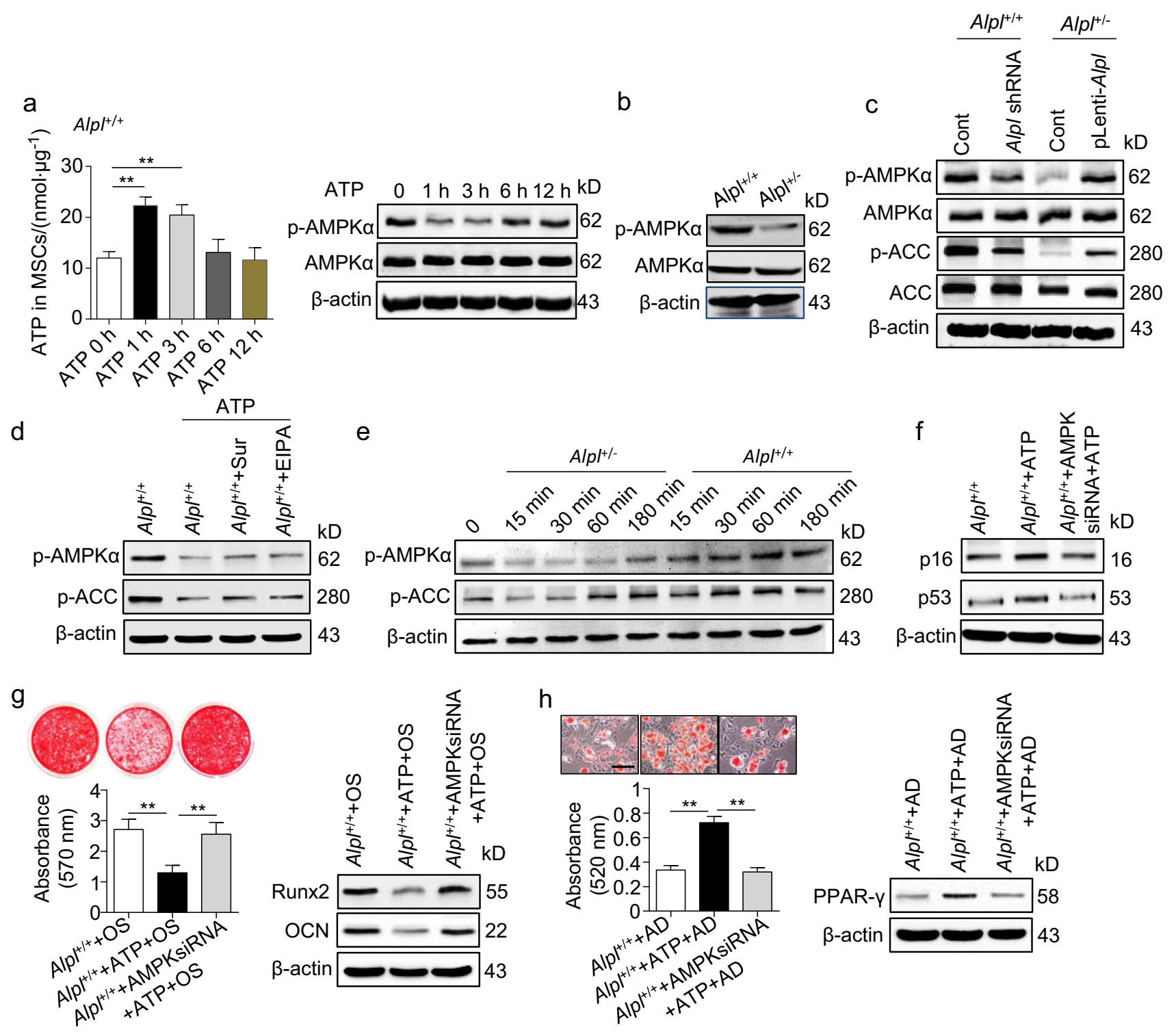

Fig. 5 ATP-mediated AMPK $\alpha$ pathway inactivation contributes to MSC dysfunction. a Alp/ ${ }^{+/+}$MSCs were treated with $10 \mu$ mol. $\mathrm{L}^{-1}$ ATP. Intracellular ATP concentrations and expression levels of AMPK $\alpha$ and p-AMPK $\alpha$ were examined 0, 1, 3, 6 and $12 \mathrm{~h}$ after treatment. $\mathbf{b}$ Expression levels of AMPK $\alpha$ and p-AMPK $\alpha$ in the $A / p I^{+/+}$and $A / p I^{+/-}$MSCs were examined by western blotting. c Expression levels of AMPK $\alpha, \mathrm{p}-\mathrm{AMPK} \alpha$, $\mathrm{ACC}$ and $\mathrm{p}-\mathrm{ACC}$ in $\mathrm{Alpl^{+/+ }}$ and $\mathrm{Alpl^{+/- }} \mathrm{MSCs}$ transfected with lentivirus were analyzed by western blotting. d Alp/ ${ }^{+/+}$MSCs were treated with $10 \mu \mathrm{mol} \cdot \mathrm{L}^{-1}$ ATP with or without $50 \mu \mathrm{mol} \cdot \mathrm{L}^{-1}$ EIPA and $100 \mu \mathrm{mol} \cdot \mathrm{L}^{-1}$ suramin, and the expression levels of $\mathrm{p}$-AMPK $\alpha$ and $\mathrm{p}$-ACC were analyzed by western blotting. e 293T cells were treated with medium of $A l p I^{+/+}$and $A / p I^{+/-} \mathrm{MSCs}$, and the expression levels of p-AMPK $\alpha$ and p-ACC were analyzed by western blotting. f-h Downregulated AMKP $\alpha$ expression in Alp/ ${ }^{+/+}$MSCs and treatment with or without $10 \mu \mathrm{mol} \cdot \mathrm{L}^{-1}$ ATP. Ageingspecific genes were analyzed at $48 \mathrm{~h}$ by western blotting. Alizarin Red and Oil Red $\mathrm{O}$ staining and quantifications were performed on day 21 and day 14 after the osteogenic/adipogenic induction (OS/AD). Expression levels of Runx2, OCN and PPAR- $\gamma$ were examined by western blotting on day 7 after induction. Scale bars, $100 \mu \mathrm{m} . n=6$ per group. The data are presented as the means $\pm s . d$. of each independent experiment performed in triplicate. ${ }^{* *} P<0.01$. One-way analysis of variance (ANOVA)

premature bone ageing characteristics was also validated in 12-month-old $\mathrm{Alpl}^{+1-}$ mice as evidenced by an increased bone formation capacity and bone mass and decreased adipogenesis and ageing-related gene expression (Supplementary Fig. 8). Taken together, reactivating the AMPKa pathway by local metformin administration rescues premature bone ageing in $\mathrm{Alpl}^{+/-}$mice probably by restoring the function of endogenous MSCs.

Alpl-controlled cell fate is also observed in human MSCs Given that Alpl expression is correlated with the ageing process of murine MSCs and subsequently affects the postnatal bone phenotype, we tested whether Alpl regulates the fate of MSCs via ATP metabolism in human MSCs (hMSCs). Two patients with
HPP caused by an ALPL loss-of-function mutation were confirmed by a clinical examination and DNA sequencing (data not shown). The HPP MSCs had fewer $\mathrm{Ki}^{+} 7^{+}$cells and LAP $2 \beta^{+}$cells than the normal controls. However, relatively more SA- $\beta$-gal ${ }^{+}$cells and $\gamma^{-}$ $\mathrm{H}_{2} \mathrm{AX}^{+}$cells and higher expression levels of $p 16$ and $p 53$ were detected in the HPP MSCs (Fig. 7a, b), which is consistent with those in the $\mathrm{Alpl}^{+/-} \mathrm{MSCs}$. Furthermore, the expression levels of CD73 and CD39 did not differ between the normal and HPP hMSCs (Fig. 7c). The elevated levels of extracellular ATP due to the ALPL deficiency were also internalized by the hMSCs and inactivated the AMPKa pathway, subsequently causing senescence in the hMSCs (Fig. 7d-h). Finally, similar to the Alp/ ${ }^{+/-} \mathrm{MSCs}$, the function of the HPP MSCs was more effectively rescued by 

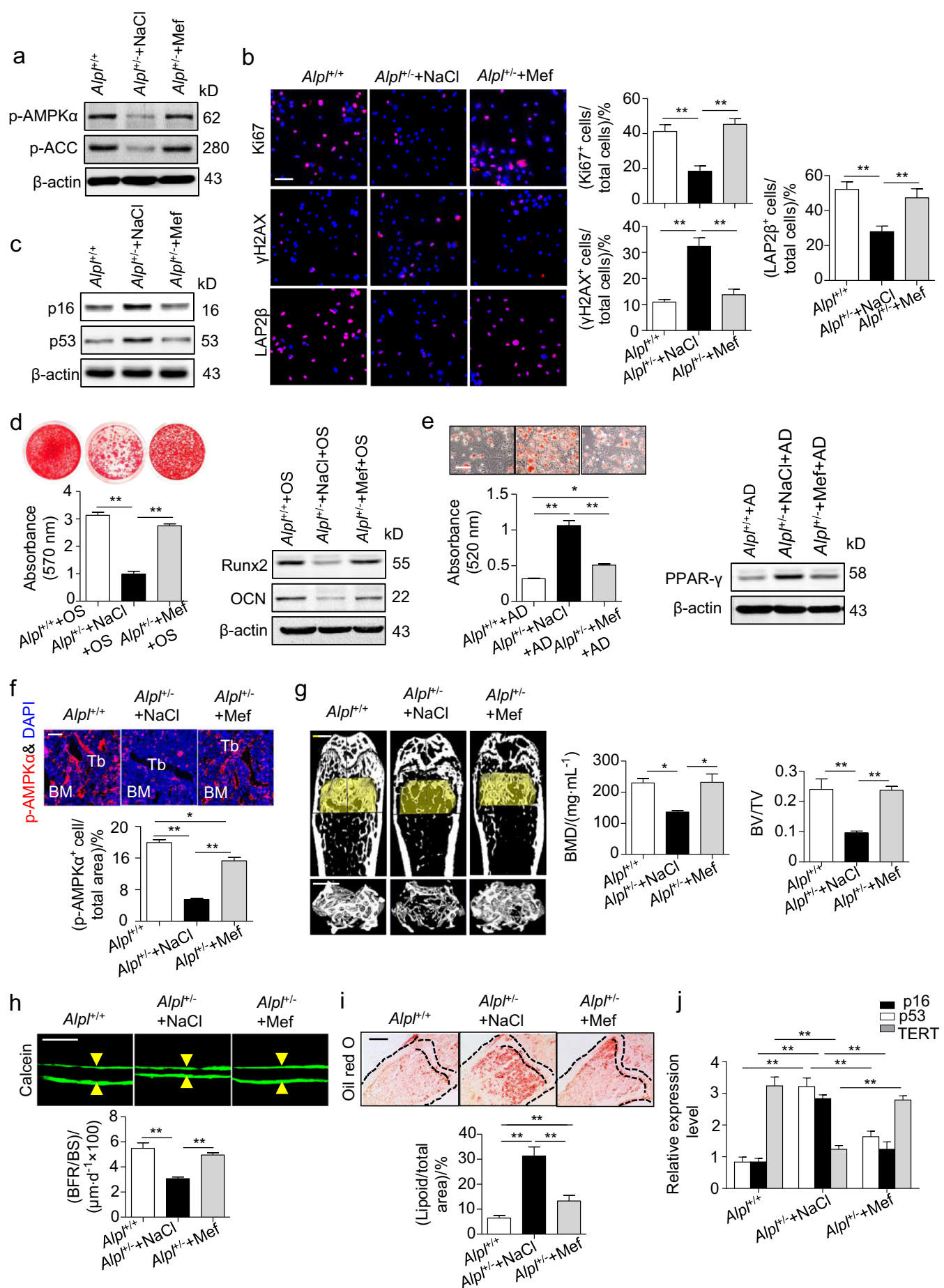

Fig. 6 Metformin treatment prevents bone ageing in $\mathrm{Alpl}^{+/-}$mice by rescuing the impaired function of $\mathrm{MSCs}$. We injected $60 \mathrm{mg} \cdot \mathrm{kg}^{-1}$ metformin into the femoral bone marrow cavity of 4-month-old $A / p I^{+/-}$mice every 2 weeks for 1 month (total of two injections), and NaCl was used as a control. a Expression levels of p-AMPK $\alpha$ and $\mathrm{p}-\mathrm{ACC}$ in MSCs from three groups were analyzed by western blotting. b Immunostaining of Ki67, $\gamma \mathrm{H} 2 \mathrm{AX}$ and LAP2 $\beta$ in MSCs from control and metformin-treated mice. Quantification of Ki67 ${ }^{+}, \gamma \mathrm{H} 2 \mathrm{AX}{ }^{+}$and $\mathrm{LAP}_{2} \beta^{+}$ is shown in the right panel. Scale bars: $50 \mu \mathrm{m}$. c Expression levels of p16 and p53 in MSCs were examined by western blotting. d Alizarin Red staining and quantification of mineralized nodules were performed on day 21 after the osteogenic induction (OS) in the MSCs from Alp ${ }^{+/+}$ and $A /\left.p\right|^{+1-}$ mice injected with $\mathrm{NaCl}$ or metformin. Expression levels of Runx2 and OCN were examined by western blotting on day 7 after induction. e Oil Red $\mathrm{O}$ staining and quantification of fat depots were performed on day 14 after the adipogenic induction (AD). Scale bars, $100 \mu \mathrm{m}$. PPAR- $\gamma$ expression was examined on day 7 after induction by western blotting. $f$ Immunostaining analysis showing the expression of $\mathrm{p}-\mathrm{AMPK} \alpha(\mathrm{red})$ and nuclear staining (blue, DAPI) in the proximal femoral diaphysis. Quantification of p-AMPK $\alpha^{+}$cells is indicated in the bottom panel. $\mathbf{g} \mu \mathrm{CT}$ images and quantification of BMD and BV/TV. Scale bars, $1 \mathrm{~mm}$. $\mathbf{h}$ Images of calcein double labeling of trabecular bone with quantification of BFR/BS. Scale bars, $50 \mu \mathrm{m}$. i Oil Red O staining images and quantitative analysis of the area of adipose tissue over the total area of the proximal femoral diaphysis. Scale bars, $500 \mu \mathrm{m}$. j Expression levels of ageing-specific genes were examined via qRT-PCR. $n=8$ per group. The data are presented as the means \pm s.d. of each independent experiment performed in triplicate. ${ }^{*} P<0.05$, ${ }^{* *} P<0.01$. One-way analysis of variance (ANOVA) 


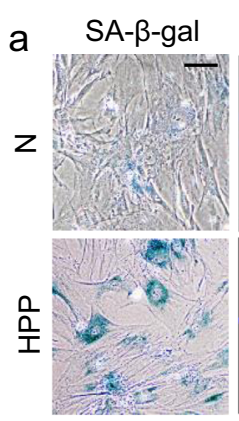

b

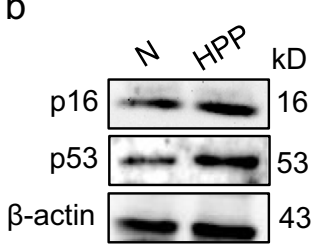

C
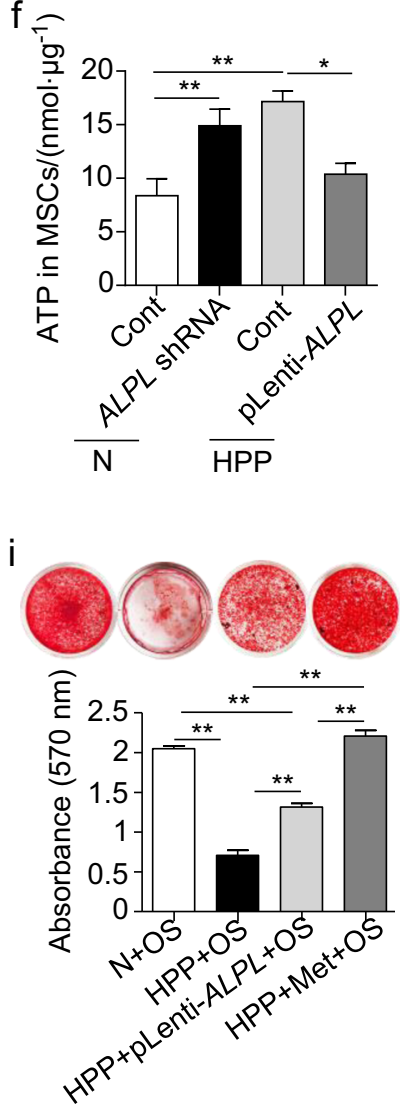

$\mathrm{yH} 2 \mathrm{AX}$
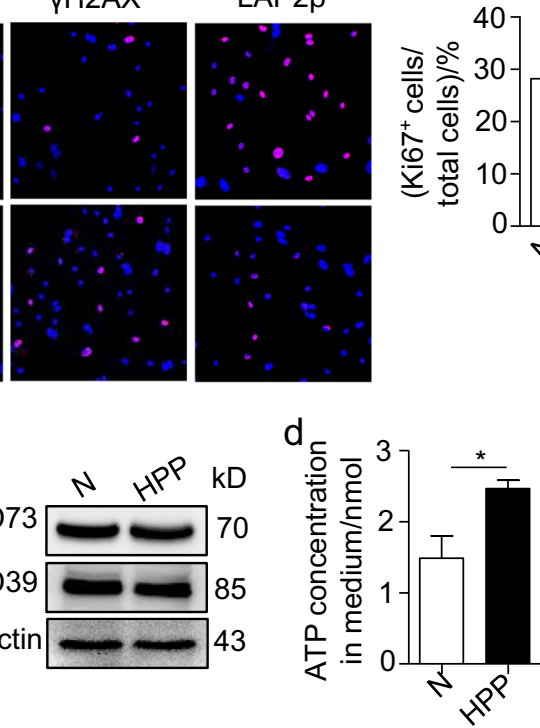
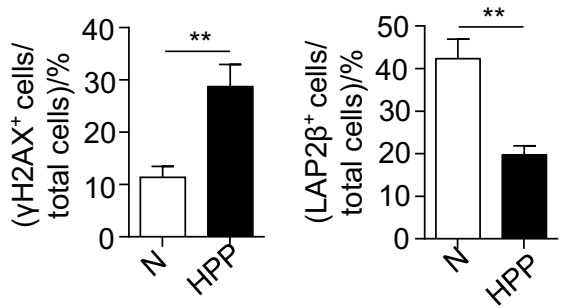

$\mathrm{e}$ 的

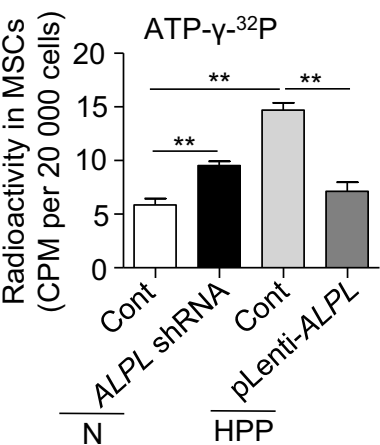

g

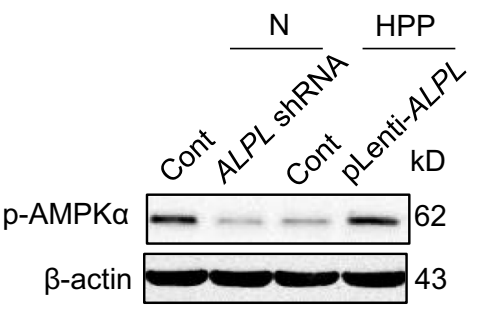

$\mathrm{h}$

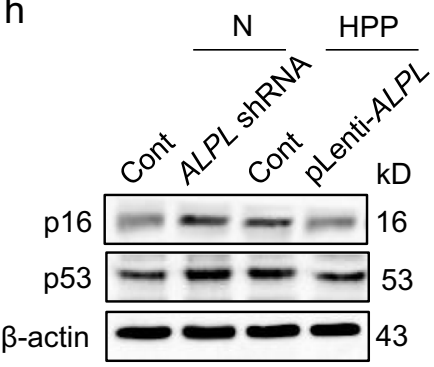

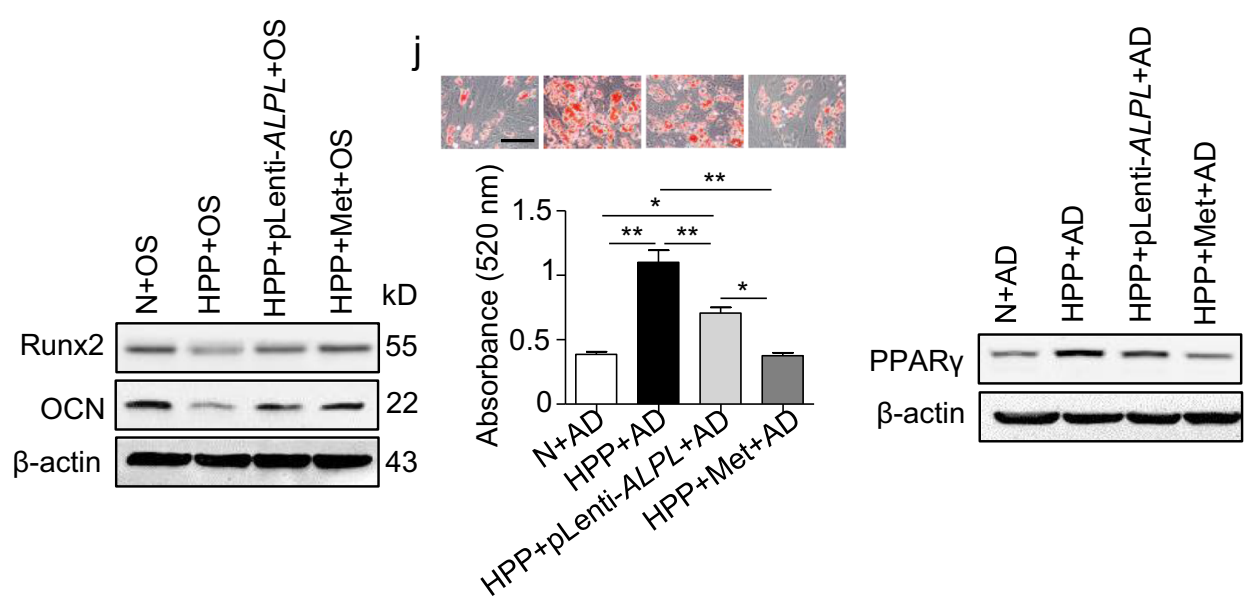

Fig. $7 \mathrm{Alpl}$ also controls the differentiation and senescence of human MSCs via ATP-mediated inactivation of the AMPK $\alpha$ pathway. a SA- $\beta$-gal staining and Ki67, $\gamma \mathrm{H} 2 \mathrm{AX}$ and LAP2 $\beta$ immunostaining of third-passage MSCs from normal controls and HPP patients. Quantification of Ki67 ${ }^{+}$, $\gamma \mathrm{H}_{2} \mathrm{AX}^{+}$and LAP2 $\beta^{+}$is indicated in the right panel. Scale bars: $50 \mu \mathrm{m}$. b Expression levels of ageing-specific genes in normal and HPP MSCs were examined by western blotting. Scale bars, $50 \mu \mathrm{m}$. c Expression levels of CD73 and CD39 in normal and HPP MSCs were examined by western blotting. d Extracellular ATP concentrations in normal and HPP MSC medium were examined by a regular ATP concentration assay. e Intracellular radioactivity was examined after a 1 - $\mathrm{h}$ treatment with ATP- $\gamma^{-32} \mathrm{P}$ in different lentiviral vector transduction groups. $\mathrm{f}$ Intracellular ATP concentrations were assayed $48 \mathrm{~h}$ after the transduction of different lentiviral vectors. $\mathrm{g}$ Western blotting analysis of $\mathrm{p}$-AMPK $\alpha$ expression in normal control and the ALPL shRNA, HPP control and pLenti-ALPL groups. $\mathbf{h}$ Expression levels of $\mathrm{p} 16$ and p53 were assayed $48 \mathrm{~h}$ after the transduction of different lentiviral vectors. i HPP MSCs overexpressing ALPL or treatment with 0.1 mM metformin, Alizarin Red staining and quantification of mineralized nodules were performed on day 28 after osteogenic induction (OS). Expression levels of Runx2 and OCN were examined by western blotting on day 7 after induction. $\mathbf{j}$ Oil Red $\mathrm{O}$ staining and quantification of fat depots were performed on day 14 after the adipogenic induction (AD). PPAR- $\gamma$ expression was examined on day 7 after induction by western blotting. Scale bars, $100 \mu \mathrm{m}$. (N) Normal control $n=5$, HPP (hypophosphatasia patient) $n=2$. The data are presented as the means \pm s.d. of each independent experiment performed in triplicate. ${ }^{*} P<0.05,{ }^{* *} P<0.01$. e-f, $\mathbf{i}$-j One-way analysis of variance (ANOVA). a, d Unpaired two-tailed Student's $t$-test. 
metformin than the function of the MSCs in the ALPL overexpression group (Fig. 7i, j). Collectively, our results indicate that Alpl is necessary for the regulation of the lineage differentiation of MSCs and prevention of their senescence.

\section{DISCUSSION}

Our study revealed that Alpl orchestrates lineage differentiation and senescence in MSCs through ATP-mediated regulation of AMPKa, thereby playing an important role in bone ageing. Specifically, Alpl deficiency results in an excessively high level of extracellular ATP due to enhanced ATP release and reduced ATP hydrolysis, which is subsequently internalized by MSCs, increasing the intracellular ATP level. This elevation inhibits the AMPKa pathway and contributes to the cell fate switch of MSCs. The reactivation of the AMPKa pathway by metformin rescues endogenous MSC function and prevents premature bone ageing in $\mathrm{Alpl}^{+/-}$mice (Supplementary Fig. 9).

Heterozygous $\mathrm{Alpl}^{+/-}$mice survive normally and exhibit a $50 \%$ decrease in TNSALP activity and expression. ${ }^{46}$ Previous studies have revealed that $A / p I^{+/-}$mice do not exhibit significant radiographic evidence of skeletal disease. ${ }^{46}$ However, we observed that the 4-month-old $\mathrm{Alp}^{+/-}$mice exhibited not only severe bone mass loss but also a marked marrow fat gain. Furthermore, the expression of the ageing-related genes $p 16$ and $p 53$ was also increased in the $A /\left.p\right|^{+/-}$mice, indicating that the Alpl deficiency led to the premature ageing of bone. A recent study revealed that a targeted deletion of $A / p /$ in both mouse OBs (Col1a1-Cre) and mesenchymal (Prx1-Cre, used to delete genes from mesenchymal progenitor cells) resulted in skeletal defects, including hypomineralization, hyperosteoidosis, rachitic changes, bone deformities and deterioration. ${ }^{47}$ Importantly, we observed that the senescent cells in the BM were mostly colocalized with MSCs rather than with mature OBs. Thus, we inferred that the Alpl deficiency-induced bone ageing phenotype may occur via the targeting of endogenous MSCs rather than mature OBs.

In bone, Alpl is localized on the entire cell surface of preosteoblasts ${ }^{48}$ and has long been used as an osteoblastic marker. ${ }^{16,49}$ MSCs are common progenitors of OBs and adipocytes in BM and abundantly express $A l p l{ }^{13}$ which is consistent with our results. However, the mechanism by which Alpl regulates the function of MSCs remains elusive. Alpl is expressed in one-cellstage embryos ${ }^{50}$ and has been proposed to ensure that ES cells are maintained in an undifferentiated state, ${ }^{51}$ suggesting that Alpl plays one or several functions in these undifferentiated cells and/ or during their multipotential differentiation. ${ }^{52}$ We observed that $\mathrm{Alp}^{I^{+-}}$MSCs exhibited enhanced adipogenic differentiation at the expense of osteogenic differentiation. In addition, the expression levels of the ageing-related genes $p 16$ and $p 53$ were increased after the induction of Alpl deficiency. More convincingly, the normal MSCs were changed into a diseased-cell phenotype after the downregulation of Alpl, whereas the function of diseased MSCs was rescued by Alpl overexpression. To further validate the role of Alpl in the fate switch of murine MSCs, we tested the characteristics of cultured MSCs from two patients with HPP. Although HPP is caused by an ALPL loss-of-function mutation, the patients are usually characterized by defective bone mineralization and a deficiency in serum and bone ALP activity. ${ }^{5-8}$ Here, the two patients displayed significant lower ALP activity and TNSALP expression both in MSCs and serum (data not shown). Moreover, the ALPL level in the hMSCs regulated their senescence and lineage differentiation, which is consistent with the observations in the $A / p I^{+/-}$MSCs. Given that $A l p l$ is localized in the cell membrane of MSCs, we discovered that Alpl is necessary for the cell fate choice of MSCs, subsequently affecting the phenotype of postnatal bone.

hMSCs have been reported to spontaneously release ATP, ${ }^{19}$ and ATP levels may dramatically increase when the microenvironment is changed. ${ }^{25,53}$ Moreover, extracellular ATP can, in turn, affect the function of MSCs, including inhibiting proliferation, ${ }^{19}$ stimulating migration $^{54}$ and regulating differentiation. ${ }^{55,56}$ Here, we found that Alpl deficiency leads to an elevated extracellular ATP concentration primarily due to increased ATP release and decreased ATP hydrolysis. This excessive extracellular ATP subsequently performs sequential important functions in MSCs, including elevating the intracellular ATP level, inactivating the AMPKa pathway and impairing cell function. However, whether and how MSCs take up extracellular ATP remain unclear. Previous studies have reported that extracellular ATP regulates cell function by activating ionotropic $\mathrm{P} 2 \mathrm{X}$ and metabotropic $\mathrm{P} 2 \mathrm{Y}$ receptors. ${ }^{39-41}$ Additionally, a recent study showed that extracellular ATP in the form of nonhydrolyzable fluorescent NHF-ATP is internalized by macropinocytosis. ${ }^{26}$ However, in this study, we observed that neither macropinocytosis nor purinergic receptor signaling could account for the intracellular ATP elevation. In contrast, the extracellular ATP directly entered the cytoplasm of MSCs as evidenced by the regular ATP and radioactive ATP- $-\mathrm{P}^{32}$ analyses. Collectively, we revealed that Alpl could simultaneously orchestrate ATP release and internalization and has an important impact on cell function.

Metformin, which is a first-line drug used for the treatment of type 2 diabetes, has been shown to successfully extend longevity and lifespan in experimental mice. ${ }^{57}$ Therefore, the use of metformin as an anti-ageing drug has been recently suggested based on its wide application in clinical practice, well-known pharmacokinetics and acceptable toxicity. ${ }^{45,57}$ In our study, the metformin treatment exhibited a stronger capacity to rescue the function of $\mathrm{Alpl}^{+/-} \mathrm{MSCs}$ both in vitro and in vivo than the recovery of the Alpl level. We believe that although the elevation in intracellular ATP was rescued by the overexpression of Alpl, the impaired intracellular pathways in the MSCs were still incompletely recovered, partially accounting for the weaker therapeutic effect. Some previous studies have reported that metformin may protect against fragility fracture. ${ }^{21}$ However, other studies have revealed that although a minor effect of metformin on bone metabolism cannot be excluded, the actions of this drug on the bone were not sufficient to modify the incidence of fractures. ${ }^{21}$ Here, we found that injections of metformin into the BM cavity could successfully rescue the impaired function of endogenous MSCs and prevent premature bone ageing in $\mathrm{Alpl}^{+/-}$mice. We believe that the different therapeutic effect compared with that reported in previous studies may be due to the murine model and different routes of metformin administration.

Collectively, we revealed a previously unrecognized role of Alpl in the prevention of bone ageing via ATP-mediated MSC functional alterations and that pathway-guided metformin treatment may provide an effective therapy for Alpl-deficient bone ageing. Further studies are needed to explore the mechanism by which Alpl regulates the ability of MSCs to release ATP. Furthermore, because many organs in the $\mathrm{Alpl}^{+/-}$mice manifested ageing characteristics (data not shown), systematic injections of metformin in $\mathrm{Alpl}^{+/-}$mice and confirmation of the changes in other organs may be needed in future experiments.

\section{MATERIALS AND METHODS}

Mice

Female $\mathrm{Alpl}^{+/+}, \mathrm{Alpl}^{+/-}$(B6.129S7-Akp2 ${ }^{\mathrm{tm} 1 \text { Sor }} / \mathrm{J}$, pure C57BL/6J genetic background) and $A / p / /^{\text {Cre/+}}$; Rosa ${ }^{26 \mathrm{mTm}^{\prime} /+}$ (Stock No. 007676) mice were purchased from Jackson Laboratories (Bar Harbor, ME, USA). Female immunocompromised nude (CAnN.CgFoxn1nu/CrlVr) 2- and 24-month-old mice were purchased from Vital River Laboratory Animal Technology Co. Ltd. (Beijing, China). Female SAMPR1 and SAMP6 mice were purchased from Peking University Laboratory Animal Center. All procedures involving animals were approved by the Animal Use and Care Committee of 
the Fourth Military Medical University (license number: SYXK 2012-0023).

Primers used for genotyping include the following: olMR0137 5'-CCGTGCATCTGCCAGTITGAGGGGA-3' - Mutant; olMR0138 - 5'CTGGCACAAAAGAGTTGGTAAGGCAG-3' - Wild-type; olMR0139 5'-GATCGGAACGTCAATTAACGTCAAT-3' - Common.

\section{ALP activity assay}

The serum and intracellular ALP activities were estimated by using a kit from NJJCBIO Company (NJJCBIO, Nanjing, China) according to the manufacturer's instruction. The final ALP activity was expressed as micromoles of $\mathrm{p}$-nitrophenol release.

The ALP activity in serum can be calculated as follows: ALP activity $\left(U \cdot L^{-1}\right)=A / V / T$, where $A$ refers to the amount of pNP generated by the samples (in $\mu \mathrm{mol}$ ); $V$ refers to the volume of sample added to the assay well (in L); and T refers to the reaction time (in minutes).

The ALP activity in cells is calculated as follows: ALP activity $\left(\mathrm{U} \cdot \mathrm{g}^{-1}\right)=\mathrm{A} / \mathrm{V} / \mathrm{T}$, where $\mathrm{A}$ refers to amount of pNP generated by the samples (in $\mu \mathrm{mol}$ ); $V$ refers to the number of samples added to the assay well (in g); and T refers to the reaction time (in minutes).

\section{Immunofluorescent staining}

The bones were fixed in $4 \%$ paraformaldehyde for $24 \mathrm{~h}$ at $4{ }^{\circ} \mathrm{C}$, decalcified in $10 \%$ EDTA (pH 7.4), embedded in or tissue-freezing medium (Leica), and sectioned into $10 \mu \mathrm{m}$ sections. For the immunofluorescence assay, heat-induced antigen retrieval was performed with sodium citrate buffer $\left(10 \mathrm{mmol} \cdot \mathrm{L}^{-1}\right.$ sodium citrate, $0.05 \%$ Tween $20, \mathrm{pH} 6.0$ ) before the bone sections were blocked with $10 \%$ normal serum containing $1 \%$ bovine serum albumin (BSA) in Tris-buffered saline and Tween 20 (TBST) (pH 7.6) for $2 \mathrm{~h}$ at room temperature; then, the sections were incubated overnight at $4{ }^{\circ} \mathrm{C}$ with primary antibodies against Sca-1/Ly6A/E (Abcam, Cambridge, MA, USA, 1:100), p16 ${ }^{\text {INK4a }}$ (Abcam, 1:100), OCN (Santa Cruz, Texas, CA, USA, 1:50), PPAR- $\gamma$ (Abcam, Cambridge, MA, USA, 1:50) and phospho-AMPKa (Cell Signaling, Boston, MA, USA, 1:50) for $2 \mathrm{~h}$ and subsequently incubated with secondary antibodies. The positive cells were examined under a laser scanning confocal microscope (Olympus FluoViem FV 1000, Tokyo, Japan). The quantitative histomorphometric analysis was conducted with Image-Pro Plus software.

Immunohistochemistry analysis

At the time of sacrifice, the femora were resected and fixed in $4 \%$ paraformaldehyde, decalcified with 10\% EDTA (pH 7.0) and embedded in paraffin. Then, $6-\mu \mathrm{m}$ thick longitudinal sections were prepared for TNSALP immunohistochemistry. We incubated the sections with a primary antibody against mouse TNSALP (R\&D Systems, Minneapolis, MN, USA, 1:100) overnight at $4{ }^{\circ} \mathrm{C}$ and subsequently with secondary antibodies. The detection of the immunoreaction was achieved using a streptavidin-horse radish peroxidase system (Dako, Carpinteria, CA, USA). The quantitative histomorphometric analysis was conducted with Image-Pro Plus software.

Senescence-associated $\beta$-galactosidase staining

The femora were resected and fixed in $4 \%$ paraformaldehyde, decalcified with $10 \%$ EDTA ( $\mathrm{pH} 7.0$ ) and embedded in paraffin. Then, 6- $\mu \mathrm{m}$ thick longitudinal sections and MSCs seeded in a 24well plate for $24 \mathrm{~h}$ were prepared for the SA- $\beta$-gal staining. We used a staining kit (Cell Signaling). Briefly, we collected the bone slices and MSCs and then rinsed the sample once with $1 \times$ phosphate-buffered saline (PBS; $2 \mathrm{~mL}$ or a $35 \mathrm{~mm}$ well plate or matched volume of media). Then, $1 \mathrm{~mL}$ of $1 \times$ Fixative Solution was added to each $35 \mathrm{~mm}$ well, and the samples were allowed to fix for 10-15 min at room temperature. The samples were rinsed twice with $1 \times$ PBS. Then, $1 \mathrm{~mL} \beta$-gal staining solution was added to each $35 \mathrm{~mm}$ well, and the sample was incubated at $37^{\circ} \mathrm{C}$ at least overnight in a dry incubator. The SA- $\beta-$ gal ${ }^{+}$cells were stained blue under a microscope. The samples were analyzed by ImagePro Plus software.

\section{$\mu \mathrm{CT}$ analysis}

The mice femora were scanned with an Inveon $\mu C T$ system (Siemens AG, Germany). The cross-sectional volumetric bone mineral density (BMD) was measured at the right femur middiaphysis. Using two-dimensional images, a region of interest in the secondary spongiosa was manually drawn near the endocortical surface, and cancellous bone morphometric parameters, including the BMD and BV/TV, were assessed.

\section{Oil red $\mathrm{O}$ staining}

To assess the fat tissue in the trabecular areas, the mice femora were fixed in $4 \%$ paraformaldehyde and decalcified with $10 \%$ EDTA ( $\mathrm{pH} 7.0$ ), followed by cryosectioning. Then, $10-\mu \mathrm{m}$ thick sections were stained with Oil Red $O$ for 10-20 min at room temperature, and the positive areas were quantified under a microscope and shown as a percentage of the total area. Then, the sections were washed with $60 \%$ isopropanol and twice with PBS. All reagents used for the Oil Red $O$ staining were purchased from Sigma-Aldrich (St. Louis, MO, USA).

Bone histomorphometric analysis

To examine the new bone formation rate, the mice received a double injection of calcein intraperitoneally $\left(10 \mathrm{mg} \cdot \mathrm{kg}^{-1}\right.$ body weight; Sigma) $14 \mathrm{~d}$ and $2 \mathrm{~d}$ prior to euthanasia. The tibiae and femora were isolated, fixed in $95 \%$ ethanol, and embedded in methyl methacrylate. A microtome was used to prepare $50-\mu \mathrm{m}$ longitudinal sections. The bone dynamic histomorphometric analyses of BFR/BS were performed using Image-Pro Plus software under a fluorescence microscope (Leica, Wetzlar, Germany, \#DMI6000B).

\section{TRAP staining}

To perform the TRAP staining, decalcified femora sections were fixed with a mixture of $3 \%$ formaldehyde, $67 \%$ acetone and $25 \%$ citrate solution and stained for TRAP using a commercially available kit (Sigma). Five random fields of view per section were captured under a microscope to quantify the number of TRAP ${ }^{+}$ cells.

\section{ELISA assay}

We collected WT and $\mathrm{Alpl}^{+/-}$mouse serum and performed a CTX-1 ELISA analysis by using mouse EIA kits (BOSTER, Wuhan, China). We performed all ELISA assays according to the manufacturers' instructions.

\section{Levamisole injection in vivo}

Levamisole $\left(10 \mathrm{mg} \cdot \mathrm{kg}^{-1}\right.$ body weight dissolved in $15 \mu \mathrm{L} \mathrm{NaCl}$, Sigma) was injected into the femora bone marrow cavity of 4month-old $\mathrm{Alpl}^{+/-}$mice three times per week for 1 month. The control mice received a comparable volume of $\mathrm{NaCl}(n=8$ per group). All mice were harvested at day 30 after the injections for analysis.

Isolation of mouse BM MSCs

The mouse BM cells $\left(2 \times 10^{7}-3 \times 10^{7}\right)$ were flushed from the long bones with $3 \%$ fetal bovine serum (FBS) in PBS. A single-cell suspension of all nuclear cells was obtained by passing all BM cells through a $70-\mu \mathrm{m}$ cell strainer (Bioscience, Dümmer, Germany). Then, $2.5 \times 10^{5}$ cells per square centimeter were seeded into $10-\mathrm{cm}$ culture dishes (Corning, Lowell, MA, USA) and incubated at $37{ }^{\circ} \mathrm{C}$ in $5 \% \mathrm{CO}_{2}$. After $48 \mathrm{~h}$, the cultures were washed with PBS to eliminate the non-adherent cells. The attached cells were cultured for 10-15 d with a-modified essential medium (a-MEM; Gibco-BRL, Gaithersburg, MA, USA) supplemented with 20\% FBS (Gibco-BRL), 
$2 \mathrm{mmol} \cdot \mathrm{L}^{-1} \mathrm{~L}$-glutamine, $100 \mathrm{U} \cdot \mathrm{mL}^{-1}$ penicillin, and $100 \mathrm{mg} \cdot \mathrm{mL}^{-1}$ streptomycin (Invitrogen, Gaithersburg, MD, USA). The cell culture protocol and surface marker identification have been described in our previous studies ${ }^{16,58}$ (Supplementary Table 1).

\section{qRT-PCR analysis}

The total RNA was isolated from the cells, BM and other soft tissues by using RNAiso plus (TaKaRa, Tokyo, Japan) according to the manufacturer's instructions. The mRNA was converted to complementary DNA, and quantitative reverse transcriptase-PCR (qRT-PCR) detection was carried out by PrimeScript ${ }^{T M}$ RT master mix (TaKaRa, RR036A) and SYBR Premix Ex Taq ${ }^{T M}{ }_{I I}$ (TaKaRa). A CFX96 Trademark Real-time PCR detection system (Bio-Rad, Richmond, CA, USA) was used for the detection. The expression levels of $p 16^{I N K 4 A}, p 53$ and TERT (TaKaRa) were examined.

Flow cytometric analysis

The BM cells were flushed, collected in a-MEM with $2 \% \mathrm{FBS}$, diluted to $1 \times 10^{6}$ cells in $400 \mu \mathrm{L}$ of medium and incubated with anti-mouse CD45-APC (eBioscience, San Diego, CA, USA), CD31-PE (eBioscience), Ly-6A/E (Sca-1) FITC (eBioscience), and CD73-PE (eBioscience) antibodies for $30 \mathrm{~min}$ at $4{ }^{\circ} \mathrm{C}$. Then, the cells were sorted using fluorescence-activated cell sorting (FACS) Aria model II (BD Bioscience), and the analysis was performed with FlowJo software version 7.6.

For the identification of the cell surface markers of MSCs, $5 \times 10^{5}$ WT and $\mathrm{Alpl}^{+/-}$MSCs were incubated with Ly-6A/E (Sca-1) FITC (eBioscience), CD73-PE (eBioscience), CD90.1/Thy1.1-FITC (eBioscience), CD34-PE (Biolegend), CD45-APC (eBioscience) and TNSALP (R\&D systems) antibodies for $30 \mathrm{~min}$ on ice. The samples were analyzed using FACS Aria model II, and the analysis was performed with FlowJo software, version 7.6.

Both $1 \times 10^{6} \mathrm{WT}$ and $\mathrm{Alpl}^{+/-} \mathrm{MSCs}$ cultured for $72 \mathrm{~h}$ were collected in $1.5 \mathrm{~mL}$ Eppendorf (EP) tubes and centrifuged at $300 \times g$ for $5 \mathrm{~min}$ at room temperature. The cells were washed three times with PBS, followed by resuspension in $400 \mu \mathrm{L}$ binding buffer (eBioscience), and divided equally into two new tubes. One sample was used as a negative control, whereas $10 \mu \mathrm{L}$ Annexin VFLUOS and $10 \mu \mathrm{L}$ propidium iodide were added to the other sample, which was incubated at room temperature for $15 \mathrm{~min}$. The samples were analyzed using a FACS Aria model II, and the analysis was performed with FlowJo software version 7.6.

\section{Osteogenic and adipogenic differentiation assays}

The WT and $\mathrm{Alpl}^{+/-} \mathrm{MSC}$ s were incubated with osteogenic medium ( $100 \mathrm{nmol} \cdot \mathrm{L}^{-1}$ dexamethasone, $50 \mathrm{mg} \cdot \mathrm{mL}^{-1}$ ascorbic acid and $1 \mathrm{mmol} \cdot \mathrm{L}^{-1}$ b-glycerophosphate) (Sigma) for $21 \mathrm{~d}$ according to the manufacturer's instructions. To assess osteogenic differentiation, the cells were fixed with $60 \%$ isopropanol and stained with $1 \%$ Alizarin Red (Sigma). The expression levels of Runx2 and OCN were assayed by western blotting on day 7 after the osteogenic induction.

The WT and $\mathrm{Alpl}^{+/-} \mathrm{MSCs}$ were cultured with adipogenic medium $\left(0.5 \mathrm{mmol} \cdot \mathrm{L}^{-1}\right.$ methylisobutylxanthine, $0.5 \mathrm{mmol} \cdot \mathrm{L}^{-1}$ hydrocortisone and $60 \mathrm{mmol} \cdot \mathrm{L}^{-1}$ indomethacin; Sigma) for $14 \mathrm{~d}$. The intracellular lipid accumulation was detected by staining with Oil Red O solution. PPARY expression was assayed by western blotting on day 7 after the adipogenic induction.

In vivo bone formation assay

After culturing for $3 \mathrm{~d}$, approximately $5 \times 10^{6} \mathrm{MSC}$ were mixed with $40 \mathrm{mg}$ hydroxyapatite/tricalcium phosphate (HA/TCP) ceramic particles (Sigma) and implanted into subcutaneous pockets on the backs of 8-week-old immunocompromised mice. The control group was implanted on the other side of the same host. The implants were removed 2 months after transplantation, fixed with $4 \%$ paraformaldehyde and decalcified with buffered $10 \%$ EDTA ( $\mathrm{pH}$ 7.0). For the histological analyses, the sections were stained with hematoxylin and eosin (HE) or Masson's trichrome (BaSO Diagnostic Inc., Guangdong, China). The sections were analyzed by Image-Pro Plus software.

Western blotting analysis

The MSCs were harvested in RIPA lysis buffer (Beyotime Co., Shanghai, China). After the whole-cell protein extracts were quantified by a bicinchoninic acid (BCA) assay, the extracts were separated on NuPAGE $10 \%-12 \%$ polyacrylamide gels, transferred onto polyvinylidene fluoride (PVDF) membranes (Millipore, Billerica, $M A$, USA), blocked in 5\% BSA in TBST, and hybridized with antibodies against $\beta$-actin (Abcam, 1:4 000), TNSALP (Abcam; R\&D Systems, 1:500), p16 ${ }^{\text {INK4a }}$ (Abcam, 1:1000), p53 (Cell Signaling, 1:1 000), Runx2 (Cell Signaling, 1:1 000), OCN (Santa Cruz, 1:800), PPAR- $\gamma$ (Abcam, 1:300), AMPKa (Cell Signaling, 1:1 000), phosphoAMPKa (Thr172) (Cell Signaling, 1:1000), ACC (Cell Signaling, 1:1 000), phospho-ACC (Cell Signaling, 1:1 000), CD73 (Cell Signaling, 1:1 000) and CD39 (Proteintech, 1:800). $\beta$-Actin was used as a loading control. The signals were revealed after incubation with a secondary antibody (CABIO Biotech., Beijing, China) coupled to peroxidase by using electrochemiluminescence $(E C L)$.

Lentiviral vector construction and transduction

To construct the lentiviral vector, mouse Alpl and human $A L P L$ were amplified by PCR from human genomic DNA. The PCR product was digested with Agel and EcoRl restriction enzymes, inserted into the pLko.1 vector (Addgene), digested with BamHI and $X$ hol and inserted into the pLenti 6.3/v5-DEST vector (Addgene). The inserted fragments were verified by Sanger sequencing. A lentiviral construct containing a scrambled Alpl/ $A L P L$ sequence was used as a negative control. The lentivirus was produced by co-transfecting 293T cells with the transfer vector and two packaging vectors (i.e., psPAX2 and pMD2G). The virus was subsequently purified by ultracentrifugation. The MSCs were plated in six-well plates and transduced with the lentiviral constructs and $10 \mu \mathrm{g} \cdot \mathrm{mL}^{-1}$ polybrene (Sigma). The primers used to construct the lentiviral vectors of $A l p l$ and $A L P L$ are listed in Supplementary Table 2.

Transfection assay

siRNA duplex oligonucleotides against mouse AMPKa1/2 were obtained from Santa Cruz. Non-targeting control siRNAs (Santa Cruz) were used as negative controls. In addition, the siRNAs were transfected into the cells at a final concentration of $50 \mathrm{nM}$ using siPORT NeoFX (Invitrogen). The medium was replaced after $8 \mathrm{~h}$.

\section{PPi measurements}

The extracellular and intracellular PPi concentrations were measured by a spectrophotometric method using an EnzChek ${ }^{\circ}$ Pyrophosphate Detection Kit (Molecular Probes, Madison, USA). Briefly, we collected the culture medium or cell lysates and then added $10 \mu \mathrm{L}$ samples or standard substance into a final $100 \mu \mathrm{L}$ reaction mixture in a 96-well plate. The reaction mixture was incubated for another $30-60 \mathrm{~min}$ at $22^{\circ} \mathrm{C}$, and the absorbance was read at $360 \mathrm{~nm}$. Use a Pi-free laboratory is extremely important for processing the collected samples.

ALP inhibitor and mimic treatment

To examine the effect of TNSALP on the ATP level, we treated the WT MSCs with $100 \mu \mathrm{mol} \cdot \mathrm{L}^{-1}$ ALP inhibitor (Levamisole, Sigma) and the $\mathrm{Alpl}^{+/-} \mathrm{MSCs}$ with $1 \mathrm{U} \cdot \mathrm{mL}^{-1}$ TNSALP from porcine kidney (Sigma). The cell lysates were prepared, and all ATP levels were examined by an Enhanced ATP Assay Kit (Beyotime).

Measurement of extracellular and intracellular ATP concentration The WT and $\mathrm{Alpl}^{+/-}$cell medium and cell samples with or without lentiviral vector transfection were collected by ATP lysis buffer and centrifuged at $12000 \times g$ at $4{ }^{\circ} \mathrm{C}$ for $5 \mathrm{~min}$; then, the supernatants 
and medium of the cells were examined by an Enhanced ATP Assay Kit (Beyotime). The intracellular ATP concentration results were corrected with the total protein levels of each sample.

The WT and $\mathrm{Alpl}^{+/-} \mathrm{MSCs}$ were treated with $10 \mu \mathrm{mol} \cdot \mathrm{L}^{-1}$ ATP in the presence or absence of $10 \mu \mathrm{mol} \cdot \mathrm{L}^{-1}$ Oligomycin A (SelleckChem, Houston, Texas, USA), $50 \mu \mathrm{mol} \cdot \mathrm{L}^{-1}$ EIPA (APEXBIO, Houston, Texas, USA), $30 \mu \mathrm{mol} \cdot \mathrm{L}^{-1}$ PPADS (Sigma) or $100 \mu \mathrm{mol} \cdot \mathrm{L}^{-1}$ Suramin (Sigma) for $1 \mathrm{~h}$; then, the cells were collected by ATP lysis buffer and centrifuged at $12000 \times \mathrm{g}$ at $4{ }^{\circ} \mathrm{C}$ for $5 \mathrm{~min}$. The supernatants of the cells were examined by an Enhanced ATP Assay Kit (Beyotime). The intracellular ATP concentration results were corrected with the total protein levels of each sample.

To examine the effect of ATP or ATP-apyrase on the intracellular ATP level, we treated the WT MSCs with $10 \mu \mathrm{mol} \cdot \mathrm{L}^{-1}$ ATP (Sigma) and the $\mathrm{Alpl}^{+/-} \mathrm{MSCs}$ with $2 \mathrm{U} \cdot \mathrm{mL}^{-1}$ ATP-apyrase (Sigma). The cell lysates were prepared, and all ATP concentration examinations were performed according to the manufacturer's instructions. The results were corrected with the total protein levels of each sample.

Radioactive ATP assay

According to the experimental design, the cells were treated with $10 \mu \mathrm{mol} \cdot \mathrm{L}^{-1}$ ATP, which contained $2 \mu \mathrm{Ci}$ ATP $-\gamma^{-}{ }^{32} \mathrm{P}$ (3000 Ci per mmol, BLU002A250UC, PerkinElmer, Waltham, MA, USA), and reacted at $37{ }^{\circ} \mathrm{C}$ in $5 \% \mathrm{CO}_{2}$ for $1 \mathrm{~h}$. Then, the ATP solution was removed, and the cells were thoroughly washed with PBS. In addition, the cells were lysed. The samples were mixed with OptiPhase Supermix Cocktail (1200-439, PerkinElmer) and incubated for $10 \mathrm{~min}$, and the radioactive signals were examined by a Luminescence Counter (MicroBeta JET, PerkinElmer) for $60 \mathrm{~s}$. This study was performed to ascertain that ATP directly enters the cytoplasm of MSCs.

\section{Metformin treatment in vitro}

In total, $3 \times 10^{5} \mathrm{MSCs}$ were plated into each well of a six-well plate and cultured. After the cells reached $70 \%-80 \%$ confluency, the cells were cultured in osteogenic/adipogenic differentiation medium containing $0.1 \mathrm{mmol} \cdot \mathrm{L}^{-1}$ Metformin (Sigma) as described above, and the medium was changed every $2 \mathrm{~d}$. On day 7, we harvested the cells and subjected them to western blotting assays of OB- and adipocyte-related genes. On day 21 and 14, we performed Alizarin Red and Oil Red O staining.

Metformin injection in vivo

Metformin $\left(60 \mathrm{mg} \cdot \mathrm{kg}^{-1}\right.$ body weight dissolved in $15 \mu \mathrm{L} \mathrm{NaCl}$, Sigma) was injected into the femora BM cavity of 4- and 12month-old $\mathrm{Alpl}^{+/-}$mice every 2 weeks for 1 month (total of two injections). The control mice received a comparable volume of $\mathrm{NaCl}$ ( $n=8$ per group). All mice were harvested on day 30 after the injection for analysis.

\section{HPP subjects}

Two HPP patients (male) aged 8 and 13 years were treated by the Affiliated Hospital of Fourth Military Medical University for osteodynia and missing teeth. The healthy human BM samples were collected from five teenagers aged 10-13 years (male) who underwent alveolar bone cleft repair by auto-ilium transplantation.

\section{Isolation of human BM MSCs}

The cells were purified from the BM by the Percoll density gradient centrifugation method and cultured in a-MEM supplemented with $10 \%$ FBS (Gibco-BRL), $2 \mathrm{mmol} \cdot \mathrm{L}^{-1}$ L-glutamine (Invitrogen), $100 \mathrm{U} \cdot \mathrm{mL}^{-1}$ penicillin and $100 \mathrm{mg} \cdot \mathrm{mL}^{-1}$ streptomycin (Invitrogen) at $37^{\circ} \mathrm{C}$ in $5 \% \mathrm{CO}_{2}$. The third passage MSCs were subjected to induction of osteogenic and adipogenic differentiation.

\section{Statistics}

The data are presented as the mean \pm s.d. The data were assessed for normal distribution and similar variance among the groups before further analysis. We used unpaired, two-tailed Student's $t$ tests for comparisons between two groups and one-way analysis of variance (ANOVA) with Bonferroni for multiple comparisons. All experiments were repeated more than three times, and representative experiments are shown. $P$-values $<0.05$ were considered significant. All experimental group sizes were chosen to ensure adequate statistical power despite the highly variable nature of the studies performed. No animals or samples were excluded from analysis, and the animals were randomly assigned to groups in the studies.

\section{ACKNOWLEDGEMENTS}

This work was financially supported by grants from the Nature Science Foundation of China (81620108007), National Key Research and Development Program of China (2016YFC1101400), Nature Science Foundation of China (31571532, 31601099) and National Institutes of Health, Department of Health and Human Services (R01DE017449 to S.S.).

\section{AUTHOR CONTRIBUTIONS}

W.J.L. and L.Q.Z. designed the experiments, analyzed the data, and wrote the manuscript. K.X. collected the HPP patients and normal controls. C.H.H. constructed the ageing mouse models and analyzed the data. S.Y.L. helped with the statistical design and analysis. L.L., B.L. and F.J. contributed to the experimental design. S.S. provided suggestions regarding the project and reviewed the manuscript. Y.J. developed the concept, supervised the project, conceived the experiments and revised the manuscript.

\section{ADDITIONAL INFORMATION}

The online version of this article (https://doi.org/10.1038/s41413-018-0029-4) contains supplementary material, which is available to authorized users.

Competing interests: The authors declare no competing interests.

\section{REFERENCES}

1. Grosser, P. \& Hüsler, J. Über das Vorkommen einer Glycerophosphatase in tierischen Organen. Biochem. Z. 39, 171-176 (1912).

2. Suzuki, U., Yoshimura, K. \& Takaishi, M. Uberein enzyme-phytasek das anhydrooxy-methylen-diphosphorsaure spaltet. Bull. Coll. Agric. 7, 503-512 (1907).

3. Bolger, J. T. Heterotopic bone formation and alkaline phosphatase. Arch. Phys. Med. Rehabil. 56, 36-39 (1975).

4. Garnero, P. Biomarkers for osteoporosis management: utility in diagnosis, fracture risk prediction and therapy monitoring. Mol. Diagn. Ther. 12, 157-170 (2008)

5. Whyte, M. P. Hypophosphatasia and the role of alkaline phosphatase in skeletal mineralization. Endocr. Rev. 15, 439-461 (1994).

6. Mornet, E. Hypophosphatasia. Best. Pract. Res. Clin. Rheumatol. 22, 113-127 (2008).

7. Chang, C. Y. et al. Imaging findings of metabolic bone disease. Radiographics 36, 1871-1887 (2016).

8. Whyte, M. P. et al. Enzyme-replacement therapy in life-threatening hypophosphatasia. N. Engl. J. Med. 366, 904-913 (2012).

9. Li, H. J. et al. FOXP1 controls mesenchymal stem cell commitment and senescence during skeletal aging. J. Clin. Invest. 127, 1241-1253 (2017).

10. Van Hoof, V. O. \& De Broe, M. E. Interpretation and clinical significance of alkaline phosphatase isoenzyme patterns. Crit. Rev. Clin. Lab. Sci. 31, 197-293 (1994).

11. Coleman, J. E. \& Gettins, P. Alkaline phosphatase, solution structure, and mechanism. Adv. Enzymol. Relat. Areas Mol. Biol. 55, 381-452 (1983).

12. Pittenger, M. F. et al. Multilineage potential of adult human mesenchymal stem cells. Science 284, 143-147 (1999).

13. Bianco, P. et al. The meaning, the sense and the significance: translating the science of mesenchymal stem cells into medicine. Nat. Med. 19, 35-42 (2013).

14. Oh, J., Lee, Y. D. \& Wagers, A. J. Stem cell aging: mechanisms, regulators and therapeutic opportunities. Nat. Med. 20, 870-880 (2014).

15. Hahnel, A. C. et al. Two alkaline phosphatase genes are expressed during early development in the mouse embryo. Development 110, 555-564 (1990).

16. Liu, S. et al. MSC transplantation improves osteopenia via epigenetic regulation of Notch signaling in lupus. Cell. Metab. 22, 606-618 (2015). 
17. Morikawa, S. et al. Prospective identification, isolation, and systemic transplantation of multipotent mesenchymal stem cells in murine bone marrow. J. Exp. Med. 206, 2483-2496 (2009).

18. Sacchetti, B. et al. Self-renewing osteoprogenitors in bone marrow sinusoids can organize a hematopoietic microenvironment. Cell 131, 324-336 (2007).

19. Coppi, E. et al. ATP modulates cell proliferation and elicits two different electrophysiological responses in human mesenchymal stem cells. Stem Cells 25, 1840-1849 (2007).

20. Zimmermann, H., Zebisch, M. \& Strater, N. Cellular function and molecular structure of ecto-nucleotidases. Purinergic. Signal. 8, 437-502 (2012).

21. Street, S. E. et al. Tissue-nonspecific alkaline phosphatase acts redundantly with PAP and NT5E to generate adenosine in the dorsal spinal cord. J. Neurosci. 33, 11314-U11104 (2013).

22. Riddle, R. C. et al. ATP release mediates fluid flow-induced proliferation of human bone marrow stromal cells. J. Bone Miner. Res. 22, 589-600 (2007).

23. Burnstock, G., Arnett, T. R. \& Orriss, I. R. Purinergic signalling in the musculoskeletal system. Purinergic. Signal. 9, 541-572 (2013).

24. Kumagai, H., Sacktor, B. \& Filburn, C. R. Purinergic regulation of cytosolic calcium and phosphoinositide metabolism in rat osteoblast-like osteosarcoma cells. J. Bone Miner. Res. 6, 697-708 (1991).

25. Noronha-Matos, J. B. \& Correia-de-Sa, P. Mesenchymal stem cells ageing: targeting the "purinome" to promote osteogenic differentiation and bone repair. $J$. Cell. Physiol. 231, 1852-1861 (2016).

26. Qian, Y. R. et al. Extracellular ATP a new player in cancer metabolism: NSCLC cells internalize ATP in vitro and in vivo using multiple endocytic mechanisms. Mol. Cancer Res. 14, 1087-1096 (2016).

27. Maj, T. et al. Oxidative stress controls regulatory $\mathrm{T}$ cell apoptosis and suppressor activity and PD-L1-blockade resistance in tumor. Nat. Immunol. 18, 1332-1341 (2017).

28. Waymire, K. G. et al. Mice lacking tissue non-specific alkaline phosphatase die from seizures due to defective metabolism of vitamin B-6. Nat. Genet. 11, 45-51 (1995).

29. Park, D. et al. Endogenous bone marrow MSCs are dynamic, fate-restricted participants in bone maintenance and regeneration. Cell. Stem. Cell. 10, 259-272 (2012).

30. Kfoury, Y. \& Scadden, D. T. Mesenchymal cell contributions to the stem cell niche. Cell. Stem. Cell. 16, 239-253 (2015).

31. Zhang, W. Q. et al. A Werner syndrome stem cell model unveils heterochromatin alterations as a driver of human aging. Science 348, 1160-1168 (2015).

32. Rezende, A. A. et al. Phosphodiesterase activity is a novel property of alkaline phosphatase from osseous plate. Biochem. J. 301(Pt 2), 517-522 (1994).

33. Ciancaglini, P. et al. Kinetic analysis of substrate utilization by native and TNAP-, NPP1-, or PHOSPHO1-deficient matrix vesicles. J. Bone Miner. Res. 25, 716-723 (2010).

34. Ohkubo, S., Kimura, J. \& Matsuoka, I. Ecto-alkaline phosphatase in NG108-15 cells: a key enzyme mediating P1 antagonist-sensitive ATP response. Br. J. Pharmacol. 131, 1667-1672 (2000)

35. Cavaliere, F., Donno, C. \& D'Ambrosi, N. Purinergic signaling: a common pathway for neural and mesenchymal stem cell maintenance and differentiation. Front. Cell. Neurosci. 9, 211 (2015).

36. Atkinson, B. et al. Ecto-nucleotidases of the CD39/NTPDase family modulate platelet activation and thrombus formation: potential as therapeutic targets. Blood. Cells Mol. Dis. 36, 217-222 (2006).

37. Commisso, C. et al. Macropinocytosis of protein is an amino acid supply route in Ras-transformed cells. Nature 497, 633-637 (2013).

38. Ivanov, A. I. Pharmacological inhibition of endocytic pathways: is it specific enough to be useful? Methods Mol. Biol. 440, 15-33 (2008).

39. Choi, I. S. et al. Enzymatic conversion of ATP to adenosine contributes to ATPinduced inhibition of glutamate release in rat medullary dorsal horn neurons. Neuropharmacology 93, 94-102 (2015).

40. Bowler, W. B. et al. Extracellular nucleotide signaling: a mechanism for integrating local and systemic responses in the activation of bone remodeling. Bone $\mathbf{2 8}$, 507-512 (2001).
41. Gallagher, J. A. ATP P2 receptors and regulation of bone effector cells. J. Musculoskelet. Neuron. Interact. 4, 125-127 (2004).

42. Hardie, D. G., Schaffer, B. E. \& Brunet, A. AMPK: an energy-sensing pathway with multiple inputs and outputs. Trends Cell Biol. 26, 190-201 (2016).

43. Chen, S. C. et al. Metformin suppresses adipogenesis through both AMP-activated protein kinase (AMPK)-dependent and AMPK-independent mechanisms (vol 440, pg 57, 2017). Mol. Cell. Endocrinol. 443, 176-176 (2017).

44. Jeyabalan, J. et al. AMP-activated protein kinase pathway and bone metabolism. J. Endocrinol. 212, 277-290 (2012).

45. Podhorecka, M., Ibanez, B. \& Dmoszynska, A. Metformin - its potential anti-cancer and anti-aging effects. Post. Hig. Med. Dosw. (Online). 71, 170-175 (2017).

46. Fedde, K. N. et al. Alkaline phosphatase knock-out mice recapitulate the metabolic and skeletal defects of infantile hypophosphatasia. J. Bone Miner. Res. 14 2015-2026 (1999).

47. Foster, B. L. et al. Conditional Alpl ablation phenocopies dental defects of hypophosphatasia. J. Dent. Res. 96, 81-91 (2017).

48. Buchet, R., Millan, J. L. \& Magne, D. Multisystemic functions of alkaline phosphatases. Methods Mol. Biol. 1053, 27-51 (2013).

49. Liu, W. et al. TCF3, a novel positive regulator of osteogenesis, plays a crucial role in miR-17 modulating the diverse effect of canonical Wnt signaling in different microenvironments. Cell Death Dis. 4, e539 (2013).

50. Merchant-Larios, H., Mendlovic, F. \& Alvarez-Buylla, A. Characterization of alkaline phosphatase from primordial germ cells and ontogenesis of this enzyme in the mouse. Differentiation 29, 145-151 (1985).

51. Millan J. L. Mammalian Alkaline Phosphatases: From Biology to Applications in Medicine and Biotechnology [M]. p.105-160 (Wiley-VCH, Weinheim, New York, 2006).

52. Esteve, D. et al. Multiple functions of MSCA-1/TNAP in adult mesenchymal progenitor/stromal cells. Stem Cells Inter. 2016, 1815982 (2016).

53. Berlier, J. L. et al. Adenosine triphosphate prevents serum deprivation-induced apoptosis in human mesenchymal stem cells via activation of the MAPK signaling pathways. Stem Cells 33, 211-218 (2015).

54. Ferrari, D. et al. Purinergic stimulation of human mesenchymal stem cells potentiates their chemotactic response to CXCL12 and increases the homing capacity and production of proinflammatory cytokines. Exp. Hematol. 39, 360-374 (2011). 374.e1-5.

55. Gharibi, B. et al. Contrasting effects of $A 1$ and $A 2 b$ adenosine receptors on adipogenesis. Int J. Obes. 36, 397-406 (2012).

56. Ciciarello, M. et al. Extracellular purines promote the differentiation of human bone marrow-derived mesenchymal stem cells to the osteogenic and adipogenic lineages. Stem Cells Dev. 22, 1097-1111 (2013).

57. Anisimov, V. N. Metformin: do we finally have an anti-aging drug? Cell Cycle (Georget., Tex.) 12, 3483-3489 (2013).

58. Liu, Y. et al. Hydrogen sulfide maintains mesenchymal stem cell function and bone homeostasis via regulation of $\mathrm{Ca}(2+)$ channel sulfhydration. Cell. Stem. Cell. 15, 66-78 (2014)

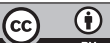

Open Access This article is licensed under a Creative Commons Attribution 4.0 International License, which permits use, sharing, adaptation, distribution and reproduction in any medium or format, as long as you give appropriate credit to the original author(s) and the source, provide a link to the Creative Commons license, and indicate if changes were made. The images or other third party material in this article are included in the article's Creative Commons license, unless indicated otherwise in a credit line to the material. If material is not included in the article's Creative Commons license and your intended use is not permitted by statutory regulation or exceeds the permitted use, you will need to obtain permission directly from the copyright holder. To view a copy of this license, visit http://creativecommons. org/licenses/by/4.0/.

(c) The Author(s) 2018 